\title{
Macrophages are critical to the maintenance of IL-13-dependent lung inflammation and fibrosis
}

\author{
LA Borthwick ${ }^{1,2,4}$, L Barron ${ }^{2,4}, \mathrm{KM} \mathrm{Hart}{ }^{2}, \mathrm{KM}$ Vannella $^{2}$, RW Thompson ${ }^{2}$, S Oland ${ }^{2}$, A Cheever ${ }^{2}$, \\ $\mathrm{J} \mathrm{Sciurba}^{2}$, TR Ramalingam ${ }^{2}$, AJ Fisher ${ }^{1,3}$ and TA Wynn ${ }^{2}$
}

The roles of macrophages in type 2-driven inflammation and fibrosis remain unclear. Here, using CD11b-diphtheria toxin receptor (DTR) transgenic mice and three models of interleukin 13 (IL-13)-dependent inflammation, fibrosis, and immunity, we show that $\mathrm{CD} 11 \mathrm{~b}^{+} \mathrm{F} 4 / 80^{+} \mathrm{Ly}_{6 \mathrm{C}}{ }^{+}$macrophages are required for the maintenance of type 2 immunity within affected tissues but not secondary lymphoid organs. Direct depletion of macrophages during the maintenance or resolution phases of secondary Schistosoma mansoni egg-induced granuloma formation caused a profound decrease in inflammation, fibrosis, and type 2 gene expression. Additional studies with CD11c-DTR and CD11b/CD11c-DTR double-transgenic mice suggested that macrophages but not dendritic cells were critical. Mechanistically, macrophage depletion impaired effector CD4 ${ }^{+}$T helper type 2 (Th2) cell homing and activation within the inflamed lung. Depletion of $\mathrm{CD}_{11 \mathrm{~b}}{ }^{+} \mathrm{F} 4 / 80^{+} \mathrm{Ly} 6 \mathrm{C}^{+}$macrophages similarly reduced house dust mite-induced allergic lung inflammation and suppressed IL-13-dependent immunity to the nematode parasite Nippostrongylus brasiliensis. Consequently, therapeutic strategies targeting macrophages offer a novel approach to ameliorate established type 2 inflammatory diseases.

\section{INTRODUCTION}

Type 2 cytokine responses are important drivers of tissue remodeling and fibrosis in many diseases, including asthma, ulcerative colitis, and chronic helminth infections. ${ }^{1-3}$ In all these diseases, the affected organs accumulate a heterogeneous population of macrophages derived from recruited monocytes and activated tissue residents whose phenotype shifts as the inflammatory response progresses. Many recent studies have cumulated in two key conclusions: that the origins and homeostatic signals for resident tissue macrophages and monocyte-derived macrophages differ fundamentally, and that monocytes are capable of developing multiple distinct phenotypes and functions when entering tissues. ${ }^{4-9}$ Macrophage depletion studies have identified no defects in systemic initiation of type 2 cytokine responses during helminth infection. ${ }^{10-12}$ However, their overall role in maintaining or resolving established interleukin 13 (IL-13)-driven inflammation and fibrosis has not been examined.
To dissect the role of macrophages in type 2 immunity in the lung, we examined three models of T helper type 2 (Th2)-associated disease, and rather than targeting a single signaling pathway or putative effector gene, we transiently depleted macrophages using CD11b-diphtheria toxin receptor (CD11b-DTR) transgenic mice treated with diphtheria toxin (DTX). ${ }^{13,14}$ This well-established model offers the advantage of selectively depleting monocytes, monocyte descendants, and most tissue macrophages independently of their phagocytic activity and without impacting $\mathrm{CD} 11 \mathrm{~b}+$ eosinophils, neutrophils, or other innate populations. ${ }^{10,13-18}$ Our primary model was intravenous (IV) challenge of antigen-primed mice with Schistosoma mansoni eggs, creating eosinophil-rich fibrotic granulomas in the lung that progress through characteristic stages. We also employed acute house dust mite (HDM)-induced allergic airway inflammation and infection with Nippostrongylus brasiliensis, a hookworm parasite that migrates destructively through the lung and resides in the small intestine before being expelled by IL-13-dependent mechanisms.

\footnotetext{
${ }^{1}$ Tissue Fibrosis and Repair Group, Institute of Cellular Medicine, Medical School, Newcastle University, Newcastle Upon Tyne, UK. ${ }^{2}$ Immunopathogenesis Section, Laboratory of Parasitic Diseases, National Institute of Allergy and Infectious Diseases, National Institutes of Health, Bethesda, Maryland, USA and ${ }^{3}$ Institute of Transplantation, Freeman Hospital, Newcastle Upon Tyne, UK. Correspondence: TA Wynn (twynn@niaid.nih.gov)

${ }^{4}$ The first two authors contributed equally to this work. 
Unexpectedly, the combined data from all three models revealed a striking decrease in type 2 -dependent inflammation and fibrosis in the lung, without impaired IL-13 responses in draining lymph nodes, regardless of when macrophages were depleted. In contrast to previous studies based on chemical or surgical injury suggesting macrophage switch from pro- to antifibrotic roles between the initiation and resolution phases of liver fibrosis, ${ }^{13,15,19}$ we show that macrophages exhibit profibrotic activity at all stages of the IL-13-driven inflammatory response in the lung. Mechanistically, we found that macrophages play a critical role in recruiting and activating effector $\mathrm{CD} 4{ }^{+}$Th2 cells in the affected tissues, likely explaining why depleting macrophages rapidly decrease IL-13-dependent lung fibrosis. An important implication of our findings is that IL-13-driven tissue inflammation and fibrosis can resolve rapidly if macrophages are rendered incapable of recruiting $\mathrm{T}$ cells and actively maintaining tissue-localized immune responses. Therefore, therapeutic strategies that deactivate macrophages or reverse their accumulation in inflamed tissues could emerge as viable targets to ameliorate progressive IL-13driven fibrosis and other diseases associated with persistent overproduction of type 2 cytokines.

\section{RESULTS}

Macrophages are critical to maintain IL-13-dependent lung inflammation and fibrosis

When injected IV, S. mansoni eggs lodge in lung capillaries and induce a vigorous eosinophil-rich granulomatous type 2 immune response that leads to activation of collagen-producing myofibroblasts, deposition of excess extracellular matrix, and IL-13-dependent fibrosis. ${ }^{20,21}$ We examined whether the induction, maintenance, or resolution of secondary granulomatous inflammation and pulmonary fibrosis induced by S. mansoni depends on macrophages.

We first confirmed peritoneal and lung macrophages from CD11b-DTR mice were sensitive to DTX-induced death in vitro and in vivo within $18 \mathrm{~h}$ (Figure 1 and Supplementary Figure $\mathbf{S 1}$ online). To determine which inflammatory cell types were directly targeted by DTX, we treated egg-primed and -challenged CD11b-DTR mice with a single dose of DTX on day 3 (D3) after challenge and $\sim 18 \mathrm{~h}$ later analyzed lung leukocyte populations. A single dose of toxin depleted $\sim 50 \%$ of $\mathrm{CD} 11 \mathrm{~b}^{+} \mathrm{F} 4 / 80^{+}$macrophages (Figure 1a,b). In contrast, the number of total lung leukocytes, eosinophils, neutrophils, or $\mathrm{T}$ cells did not immediately decrease. Macrophages outnumbered dendritic cells (DCs) by $\sim 20$-fold, and neither CD11 ${ }^{+}$ nor $\mathrm{CD} 11 \mathrm{~b}^{-} \mathrm{DCs}$ (as defined by CD11c ${ }^{+} \mathrm{MHCII}^{+} \mathrm{F} 4 / 80^{-}$ gates) were directly reduced by DTX treatment. Such incomplete but preferential depletion of macrophages, without a direct net loss of all types of $\mathrm{CD} 11 \mathrm{~b}^{+}$inflammatory leukocytes, is a useful feature of the CD11b-DTR mice and our results match those described in a variety of models. ${ }^{10,13-18}$

The immune response to $S$. mansoni eggs generates granulomas in stages. ${ }^{21}$ Innate immunity initiates granuloma formation, then between days 4 and 7 adaptive immunity amplifies the response and granulomas reach their peak size and develop a fibrotic perimeter, predominantly driven by IL-13 from Th2 cells. Inflammation is resolving by day 14 . We designed experiments to deplete macrophages during the initiation, peak, and resolution stages of a secondary type 2 inflammatory response (Figure 2a). In these studies, depleting macrophages after challenging either naive (primary) or eggsensitized (secondary) mice with live $S$. mansoni eggs reduced granuloma volume on both days 4 and 7 (Figure $\mathbf{2 b}-\mathbf{d}$ and Supplementary Figure S2). Surprisingly, although macrophages are necessary for the resolution of sterile inflammation and fibrosis, ${ }^{13,19}$ depletion after the peak of the response dramatically reduced fully established granulomatous inflammation by day 14. Indeed, the granulomas measured on day 14 in DTX-treated mice were even smaller than those measured on day 4 when secondary granulomas were first forming. Total lung collagen content did not change during the first 4 days after S. mansoni egg challenge in antigen-primed mice but increased by $\sim 50 \%$ on day 7 and nearly $\sim 100 \%$ by day 14 (Figure 2e). Depletion of macrophages significantly inhibited the increase in lung collagen on days 7 and 14 as determined by both hydroxyproline content (Figure 2e) and picrosirius red staining (Figure 2f). These results demonstrate that during a secondary granulomatous response, macrophages are critically required at all time points to maintain type 2 cytokine-driven inflammation and fibrosis within the lung.

\section{Type 2 immunity in the granulomatous lung is dependent on macrophages}

IL-12, interferon- $\gamma$ (IFN- $\gamma$ ), inducible nitric oxide synthase, and IL-10 have each been shown to negatively regulate S. mansoni egg-induced type 2 pathology. ${ }^{20,22,23}$ Therefore, we examined whether the decreases in inflammation and fibrosis observed in the DTX-treated animals were a result of changes in type 1 or regulatory gene expression. Little to no change in Il12b or Ifng expression was observed and Il10 was similarly increased in both treatment groups (Figure 3a). In addition, although Nos2 exhibited antifibrotic activity in a related model, ${ }^{23}$ we observed decreased Nos2 expression on days 7 and 14 following DTX treatment (Figure 3a), likely because macrophages are major producers of inducible nitric oxide. Therefore, counterregulation of Th2 cytokine responses by these negative regulators could not explain why depleting macrophages reduced inflammatory pathology.

T cell-derived IL- 4 and IL-13 are the critical cytokines regulating granuloma development, whereas IL-13 functions as the key mediator of fibrosis. ${ }^{24-26}$ Therefore, we also examined whether the type 2 immune response in the lung was altered following depletion. In agreement with the marked reductions in granuloma size and fibrosis, we found that expression of type 2 cytokines (Il13, Il5) and several type 2 -inducible genes (Retnla, Chi3l3, Arg1,IL13ra2) were decreased at all time points (Figure 3b). In contrast, Arg2 expression, which is not regulated by the type 2 immune response, was unaffected. Interestingly, depletion only slightly altered expression of Il13ra1 in the lungs, whereas expression of the IL-4/IL-13inducible IL-13 decoy receptor (Il13ra2) was blunted 
a

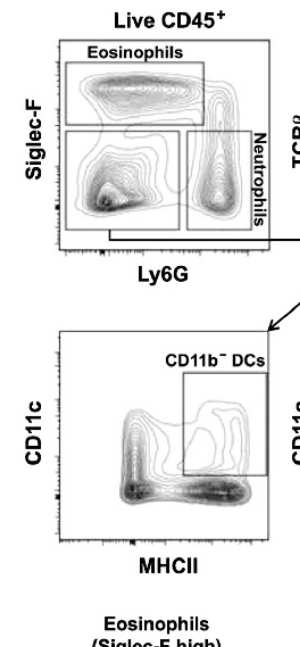

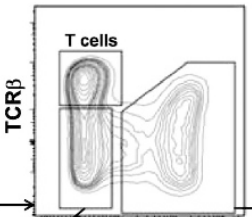
conb

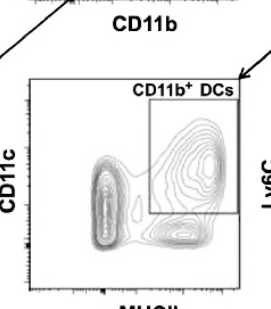

MHCII

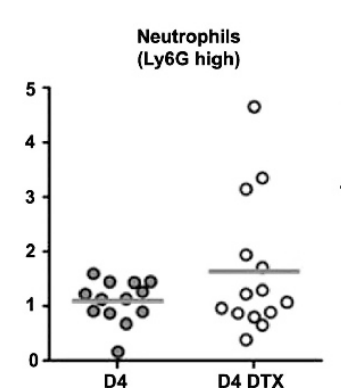

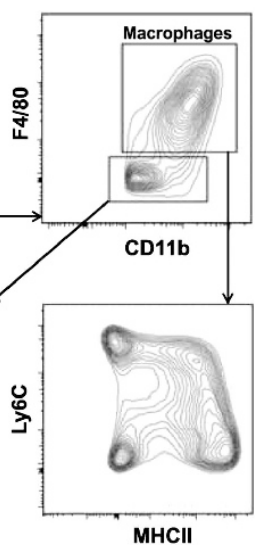

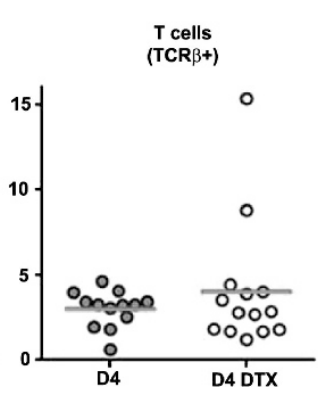

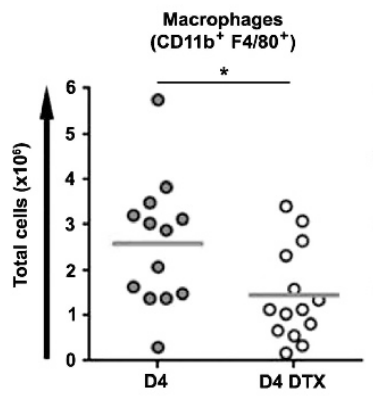
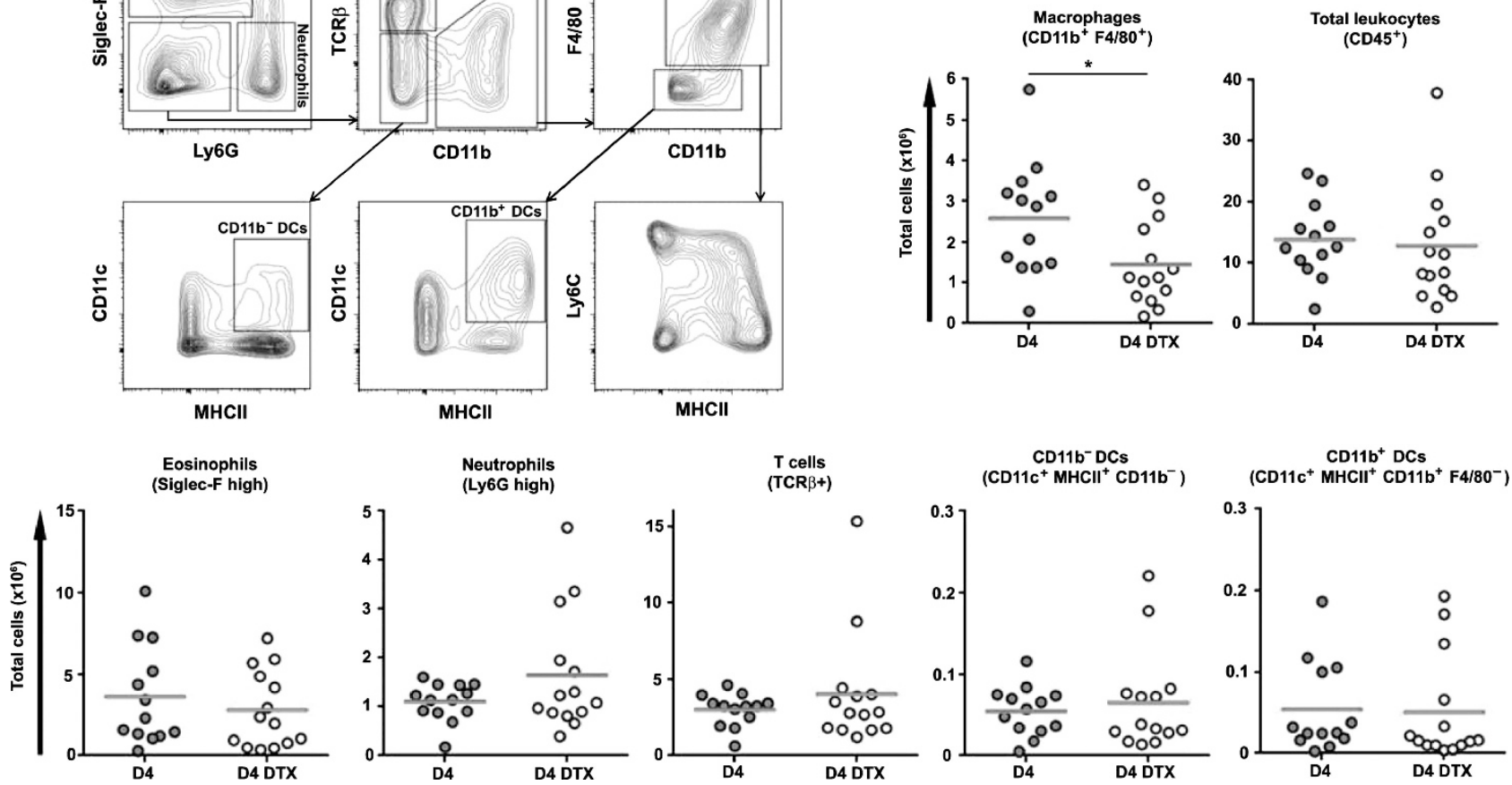

Figure 1 Diphtheria toxin (DTX) treatment of primed and challenged CD11b-diphtheria toxin receptor (DTR) mice causes direct, partial, and selective depletion of $\mathrm{CD} 11 \mathrm{~b}^{+} \mathrm{F} 4 / 80^{+}$macrophages within the granulomatous lung. CD11b-DTR egg-primed and -challenged mice were treated with DTX ( $n=14)$ or left untreated ( $n=13$ ) on day 3 (D3) and harvested on day 4 (D4). Lung leukocytes were analyzed by flow cytometry to identify directly depleted cell types. (a) Representative flow cytometry plots of untreated D4 lung leukocytes (live $\mathrm{CD} 45^{+}$) showing the gating strategy used to distinguish eosinophils (Siglec- $F^{+}$Ly6G- $/$low), neutrophils (Siglec- $F^{-}$Ly6Ghigh), T cells (TCR $\beta^{+}$), macrophages $\left(F 4 / 80^{+} \mathrm{CD}_{11 b^{+}}\right.$), as well as CD11b ${ }^{-}$and

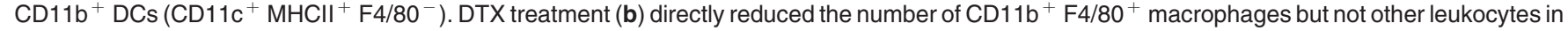
the lung. Statistical significance was calculated using unpaired two-tailed Student's $t$-test. ${ }^{*} P<0.05$. Results represent three independent experiments. DC, dendritic cell.

substantially, further supporting a marked reduction in type 2 immunity against $S$. mansoni eggs (Figure $3 \mathbf{b}$ ). Moreover, depletion caused similar changes to the pattern of gene expression in the lungs of mice challenged with eggs without priming (Supplementary Figure S3).

\section{Depletion of macrophages in the granulomatous lung indirectly reduces inflammation}

The pattern of Th2-induced gene expression in the lungs reaches a maximum between days 4 and 7 , as granuloma formation peaks. DTX treatment notably impaired this second step of $\mathrm{T}$ cell-driven amplification (Figure $\mathbf{3 b}$ ). Therefore, we next treated egg-sensitized and -challenged mice with DTX on days 3,4 , and 5 after challenge and analyzed changes in lung inflammation on day 7. Between days 4 and 7, the direct depletion of macrophages indirectly and cumulatively reduced the number of lung leukocytes in general, including eosinophils, $\mathrm{T}$ cells, and both CD11b ${ }^{+}$and CD11b ${ }^{-}$DCs, but with the exception of neutrophils (Figure 4a). This overall reduction in inflammation was accompanied by a dramatic change in the phenotype of the remaining lung macrophages (Figure $4 \mathrm{~b}, \mathrm{c})$. In control mice the fraction of $\mathrm{Ly}_{6} \mathrm{C}^{+}$macrophages, likely recently recruited monocytes, diminished between days 4 and 7, whereas expression of CD64, CD11c, and major histocompatibility complex II (MHCII), and the total number of macrophages, were maintained. In DTXtreated mice, the remaining population of $\mathrm{CD} 11 \mathrm{~b}^{+} \mathrm{F} 4 / 80^{+}$ macrophages expressed high levels of Ly6C and CD64 but little CD11c or MHCII, suggesting that new monocytes quantitatively but not qualitatively replenished depleted lung macrophages within 2 days. Together, these data (Figures 1 and 4 ) show that DTX caused direct but incomplete depletion of $\mathrm{CD} 11 \mathrm{~b}^{+} \mathrm{F} 4 / 80^{+}$macrophages in the lung without reducing other leukocyte populations. Yet, even partial macrophage depletion was sufficient to indirectly and cumulatively reduce overall lung inflammation within 3 days corresponding with the substantial loss of CD11c- and MHCII-expressing F4/80 ${ }^{+}$ cells and, within 2 days of the final DTX treatment, their replacement by cells resembling conventional monocytes.

\section{Reduced local but not systemic CD4 ${ }^{+}$Th2 cell responses} underlie decreased lung inflammation and fibrosis

As IL-13 and IL-5 were reduced in whole lung tissue (Figure 3b), we next examined Th cell activation, differentiation, and homing. Importantly, DTX treatment did not reduce the percentage (Figure 5a), number (not shown), or 
a

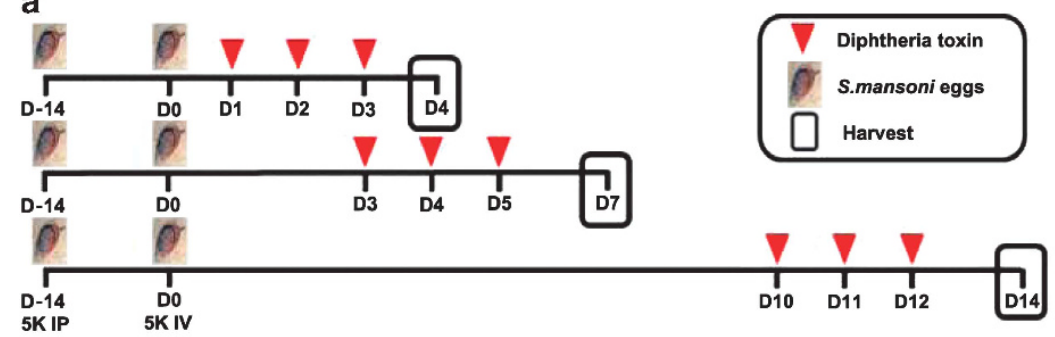

b

Secondary lung granuloma
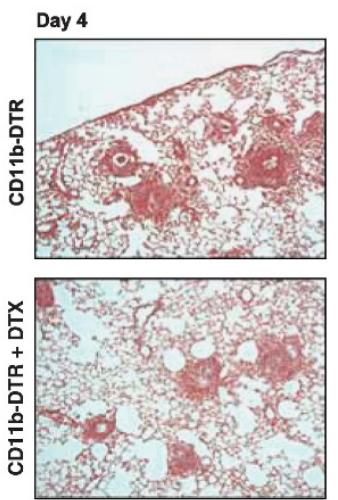

Day 7

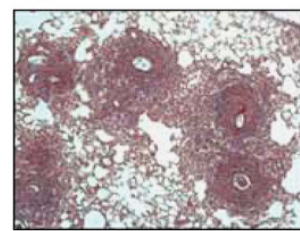

Day 14

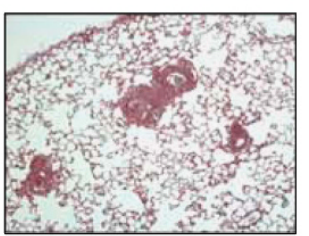

e Secondary lung granuloma
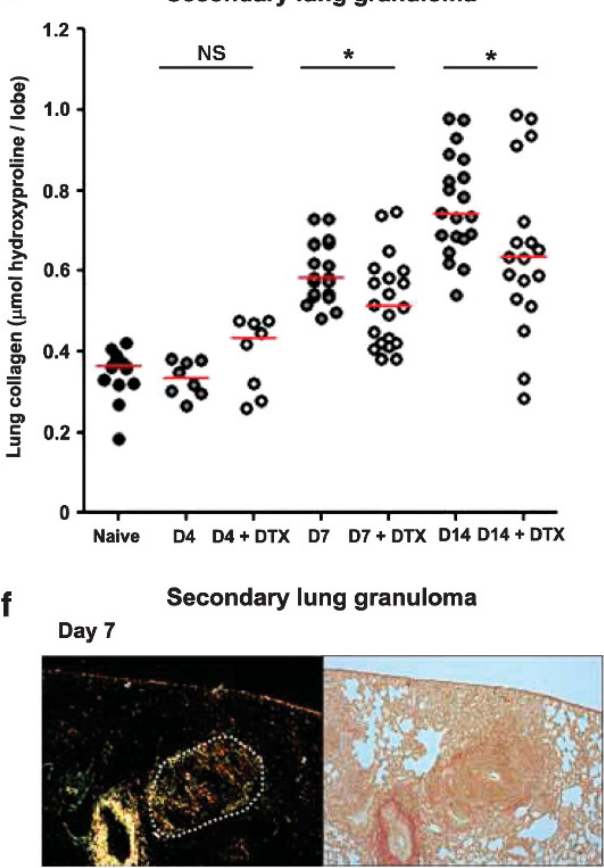

Day 7 + DTX

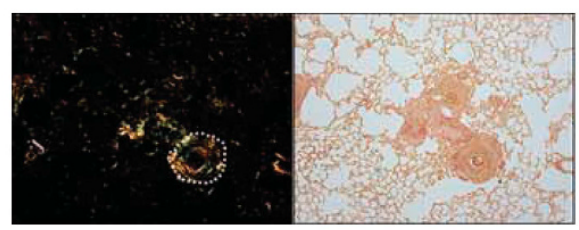

Day 14

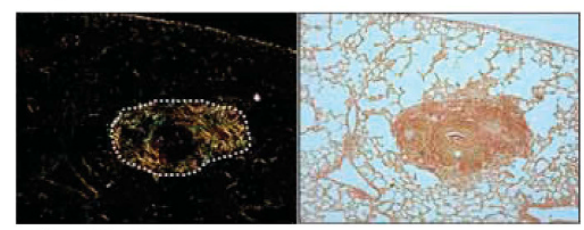

Day $14+$ DTX

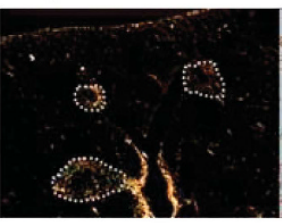

Polarized light

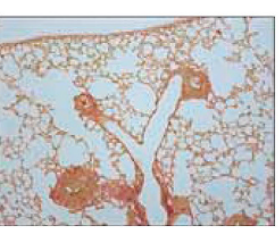

Normal light

Figure 2 Macrophages are critical promoters of both the local induction and maintenance of type 2-dependent lung fibrosis. (a) CD11b-diphtheria toxin receptor (DTR) mice were primed by intraperitoneal (IP) injection of 5,000 Schistosoma mansoni eggs on day -14 (D-14) and then challenged by a single intravenous (IV) injection of 5,000 live eggs on day 0 (D0). Mice were harvested at the initiation (D4), peak (D7), or resolution (D14) stages of lung granuloma formation. Half of the mice were treated with diphtheria toxin (DTX; $25 \mathrm{ng} \mathrm{g}^{-1}$ ) when indicated. (b) Representative images showing inflammation and collagen (blue) in Masson's trichrome-stained lung tissue from primed and challenged (CD11b-DTR) (primary lung granuloma: D4 $n=5$; D7 $n=15$; D10 $n=15$; secondary lung granuloma: D4 $n=8$; D7 $n=19$; D10 $n=19$ ), and primed, challenged, and DTX-treated (CD11b-

DTR + DTX) (primary lung granuloma: D4 $n=8 ; \mathrm{D} 7 n=18$; D10 $n=19$; secondary lung granuloma: D4 $n=7$; D7 $n=19 ;$ D10 $n=15)$ CD11b-DTR mice. Volumes of (c) primary lung granulomas (IV challenge without priming) and (d) secondary lung granulomas (IP primed, IV challenged) were scored by a pathologist blinded to groups and data presented as average granuloma volume per mouse (top panel) and individual granuloma volume (bottom panel). DTX treatment reduced both primary and secondary lung granuloma volumes at all time points assessed. (e) Change in collagen deposition in egg-primed and -challenged CD11b-DTR mice with or without DTX treatment was compared by measuring hydroxyproline content of lung tissue. (f) Collagen was visualized by picrosirius red staining of lung tissue exposed to polarized and normal transmitted light. Dotted lines outline granulomas. DTX treatment reduced lung collagen at D7 and D14. Data are presented as median and statistical significance calculated using Mann-Whitney $U$-test. ${ }^{*} P<0.05$, ${ }^{* * *} P<0.001$. Results represent two independent experiments. NS, not significant. 
a
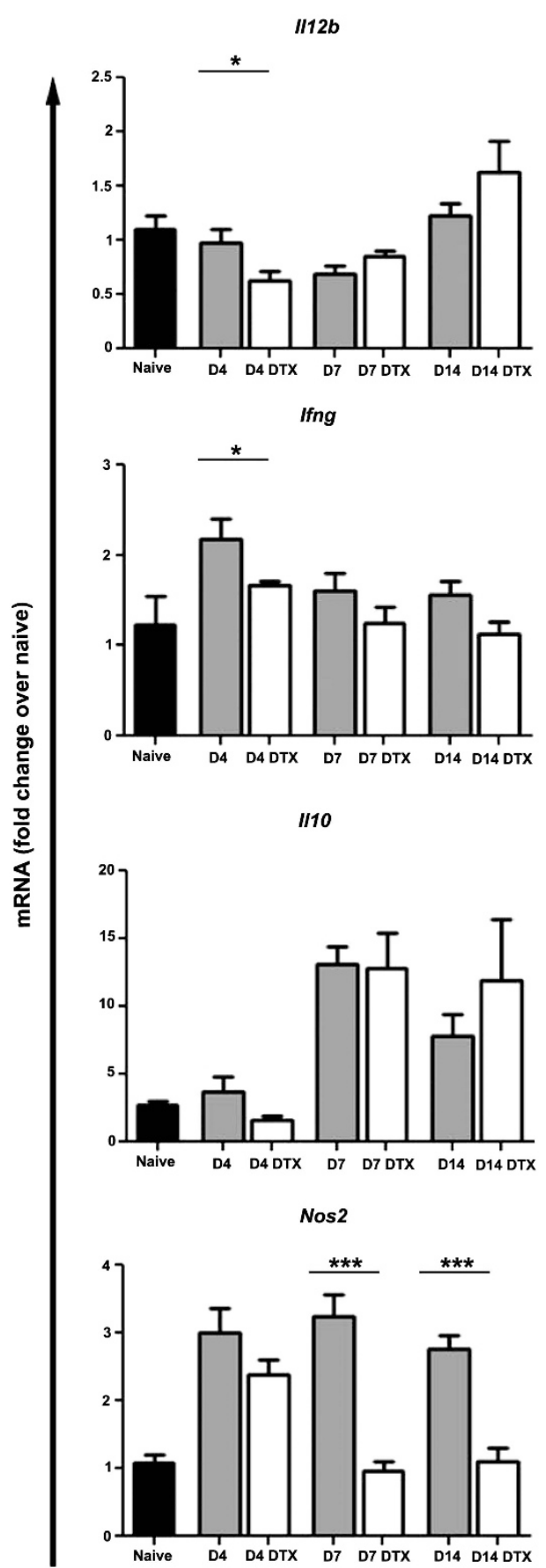

b
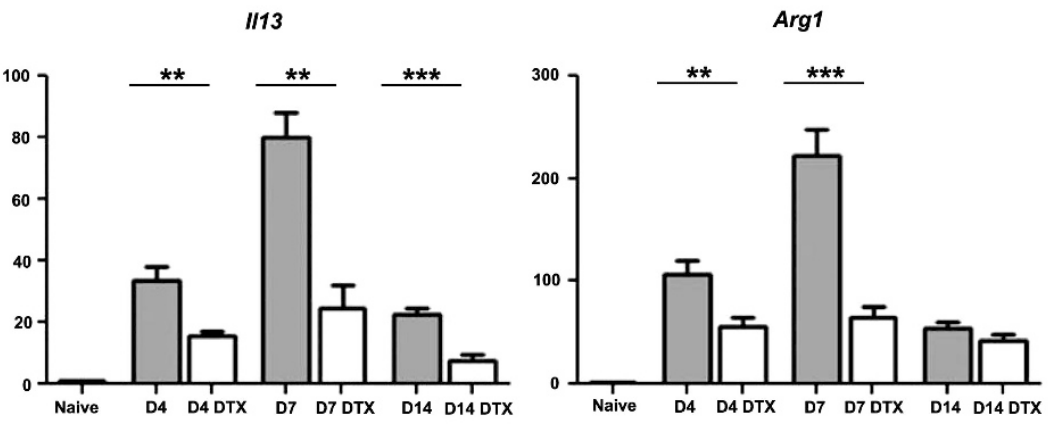

$\operatorname{Arg} 2$
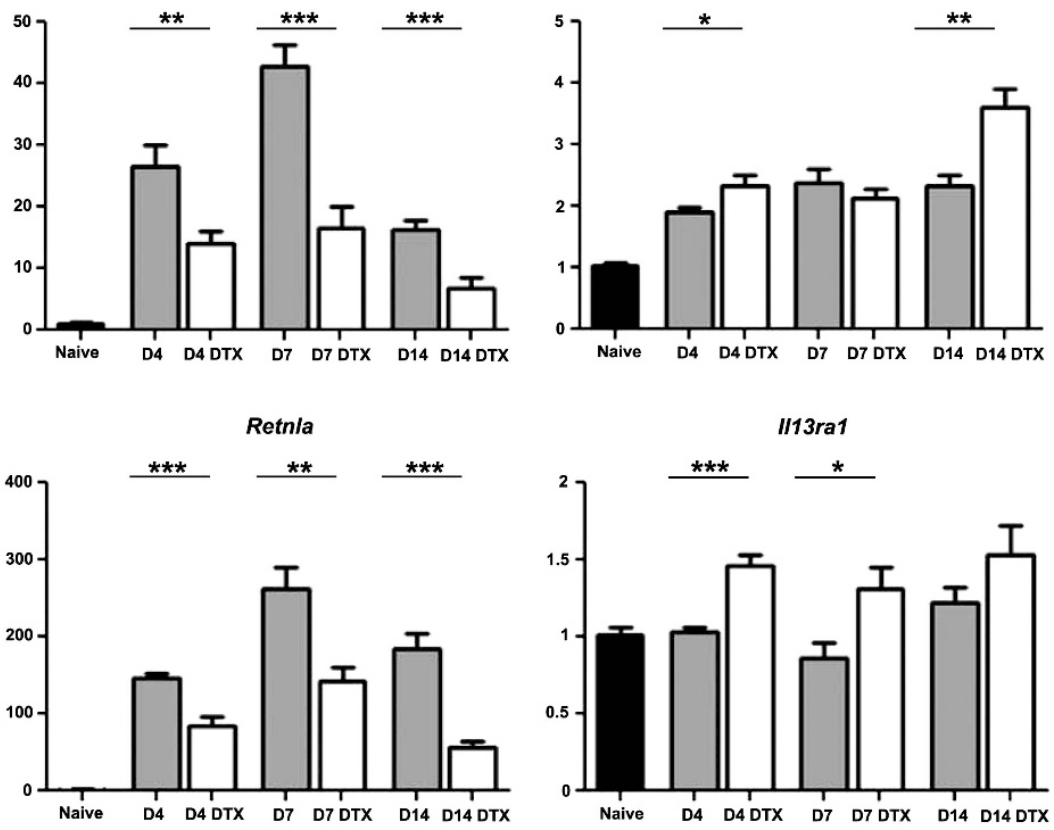

Chi3/3
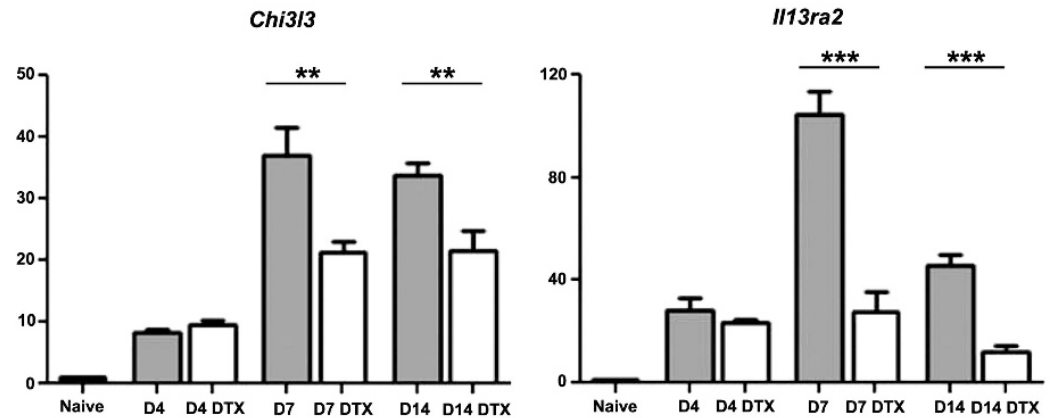

Figure 3 Type 2 immunity in the granulomatous lung is dependent on macrophages. Relative quantitative gene expression in lung tissue of naive ( $n=13$ ), egg primed and challenged (D4 $n=8 ; \mathrm{D} 7 n=9, \mathrm{D} 10 n=10$ ), and primed, challenged, and diphtheria toxin (DTX)-treated (D4 $n=8 ; \mathrm{D} 7 n=10$, D10 $n=9$ ) CD11b-diphtheria toxin receptor (DTR) mice. Results were normalized to RPLP2 and scaled to naive mice. Counter-regulatory genes (a) did not increase whereas (b) all T helper type 2 (Th2)-induced genes were more weakly induced by egg challenge following DTX treatment. Data are presented as mean \pm s.e.m. Statistical significance was calculated using unpaired two-tailed Student's $t$-test. ${ }^{*} P<0.05,{ }^{* *} P<0.01,{ }^{* * *} P<0.001$. Results represent two independent experiments. D, day.

magnitude of expression (Supplementary Figure S4) of IL-13 and IL- 4 by $\mathrm{CD}^{+}{ }^{+} \mathrm{T}$ cells in the lung-draining mediastinal lymph nodes at any time point. Instead, we found small but highly consistent decreases in the percentages and greater reduction in the numbers of IL-13- and IL-4-producing CD4 ${ }^{+}$ $\mathrm{T}$ cells in the lung, and diminished cytokine production on a per-cell basis (Figure $\mathbf{5 b} \mathbf{b}-\mathbf{d}$ ). In contrast, IFN- $\gamma$ was unchanged in either the lung or mediastinal lymph nodes, although IL-17A increased in the lymph nodes but decreased in the lung (Figure 5 and Supplementary Figure S4). Experiments with unprimed mice yielded similar results (Supplementary Figure S5, not shown). Together, these results imply that 
a

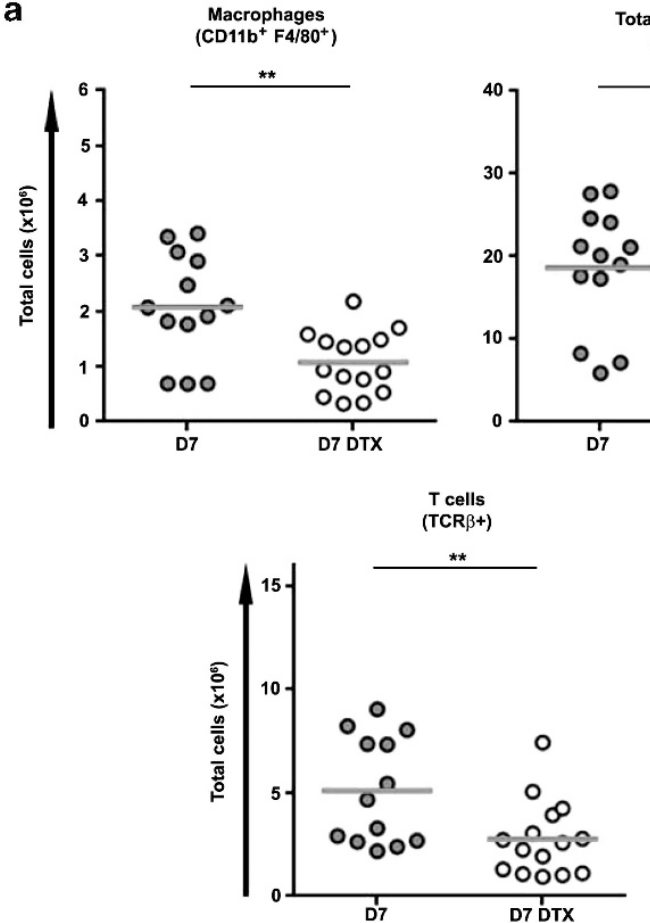

Total leukocytes (CD45')

***

b

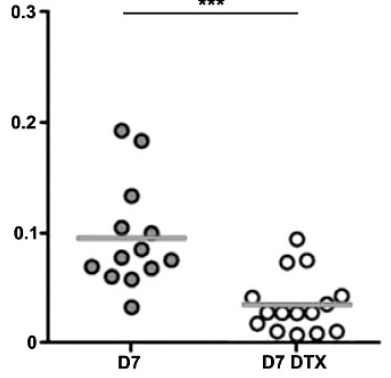

Eosinophils

(Siglec-F high)
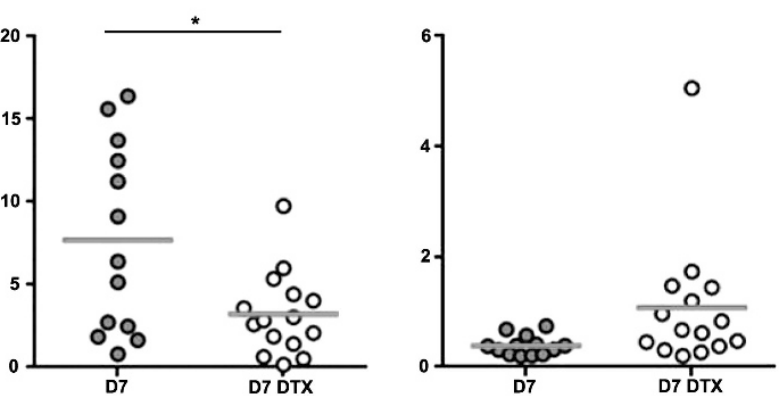

Gated on $\mathrm{CD} 11 \mathrm{~b}^{+} \mathrm{F} 4 / 80^{+}$
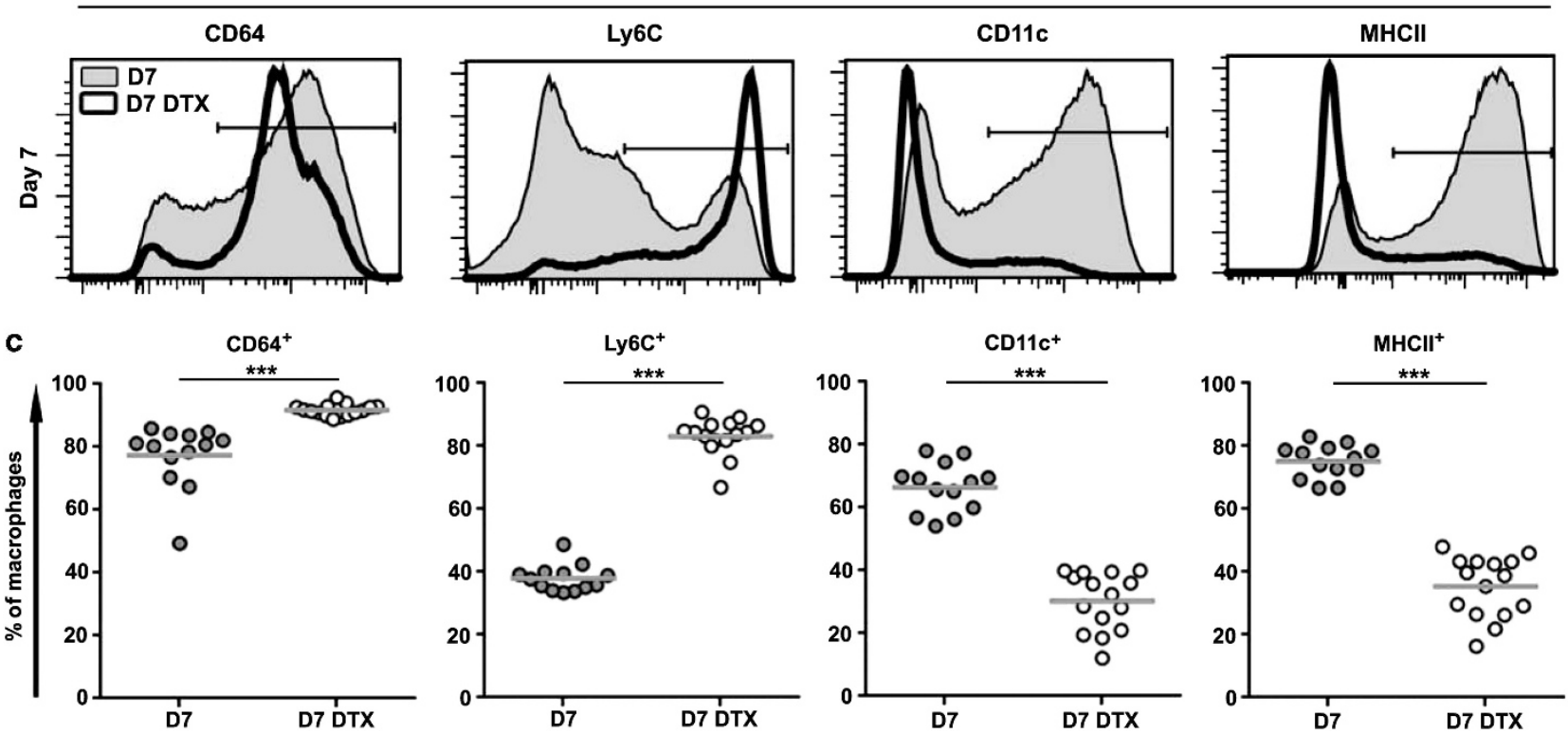

Figure 4 Direct depletion of macrophages in the granulomatous lung indirectly reduces inflammation and leads to their replacement by cells with a monocyte phenotype. CD11b-diphtheria toxin receptor (DTR) egg-primed and -challenged mice were treated with diphtheria toxin (DTX; $n=15$ ) or left untreated $(n=13)$ on days 3,4 , and 5 (D3, D4, D5) and harvested on D7. Lung leukocytes were analyzed by flow cytometry as in Figure 1 to examine the cumulative effects of depletion. The direct, partial, and selective depletion of macrophages within $18 \mathrm{~h}$ (Figure 1) led to an indirect decline in inflammatory leukocytes. (a) Net changes in lung leukocyte populations. DTX treatments reduced macrophage numbers, but also led to an indirect decline in total leukocytes, eosinophils, T cells, CD11 b ${ }^{-}$dendritic cells (DCs), and CD11 $\mathrm{c}^{+}$DCs. Neutrophils were not significantly changed. (b,c) DTX treatment shifts

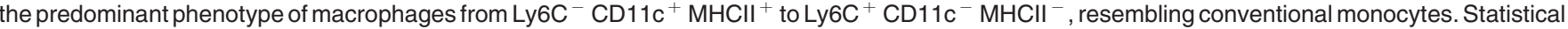
significance was calculated using unpaired two-tailed Student's $t$-test. ${ }^{\star} P<0.05,{ }^{\star \star} P<0.01,{ }^{\star \star *} P<0.001$. Results represent three independent experiments.

macrophage depletion does not interfere with T-cell priming in lymph nodes, but once the type 2 response is established macrophages play a critical role in recruiting, reactivating, and/or retaining the effector Th2 cell response in the lung.
Targeted depletion of DCs after antigen priming and egg challenge does not reduce type 2-dependent granulomas and fibrosis in the lung

The successful generation of cytokine-producing CD4 T cells in lymph nodes suggested that sufficient antigen presentation still 
a

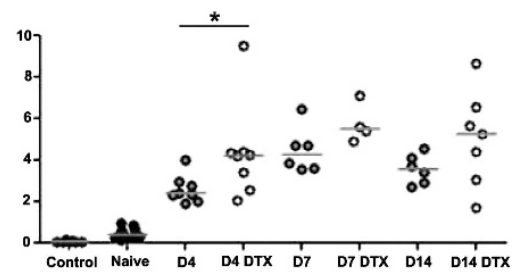

c

d
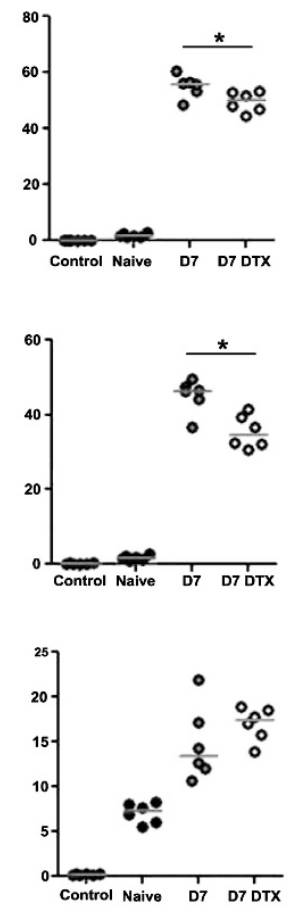

\section{1}
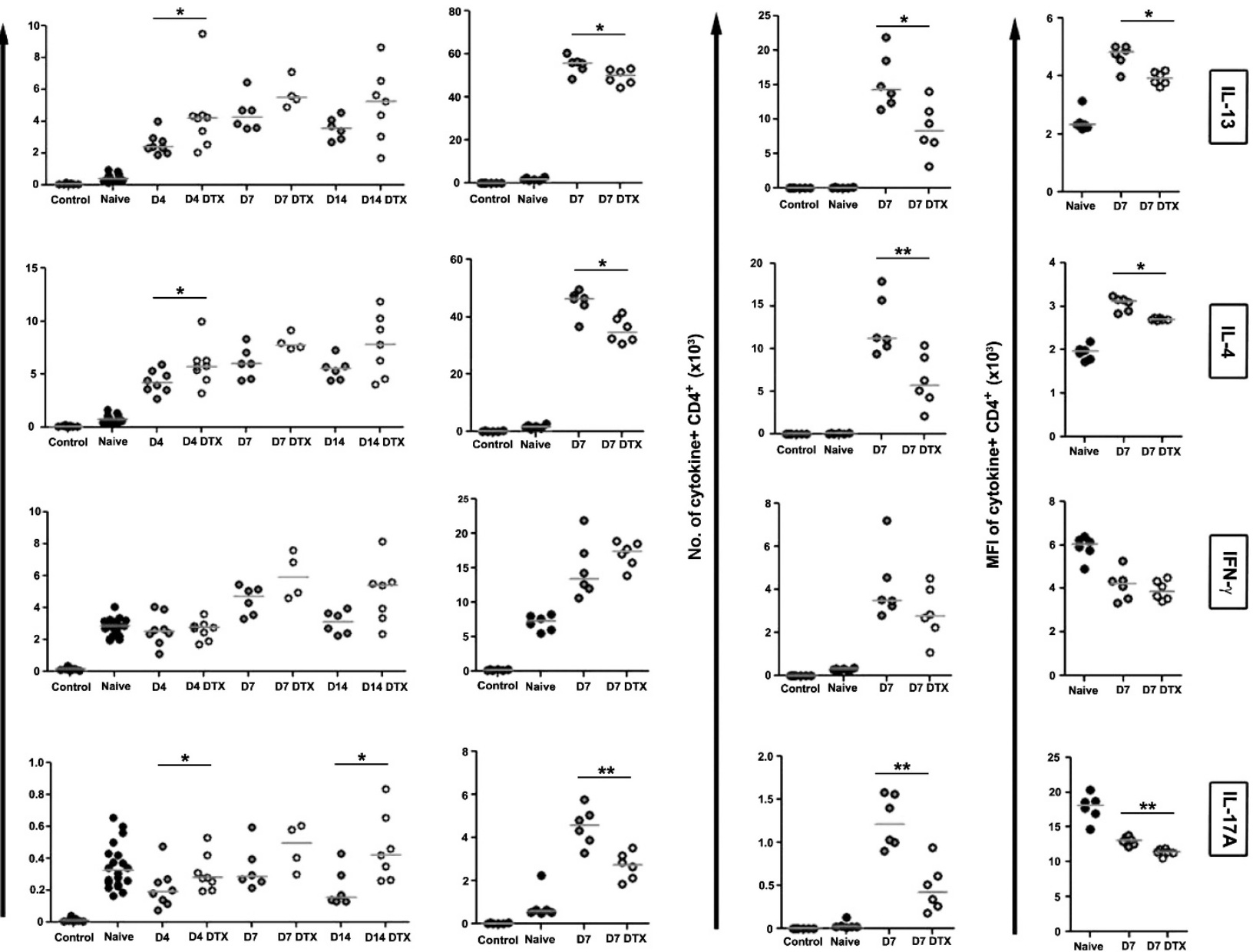

Figure 5 Reduced local but not systemic CD4 ${ }^{+}$Thelper type 2 (Th2) cell responses underlie decreased lung inflammation and fibrosis. (a) Mediastinal lymph node and (b) lung leukocytes isolated from naive (lymph node $n=20$; lung D7 $n=6$ ), egg primed and challenged (lymph node D4 $n=8$; D7 $n=6$; D10 $n=6$; lung D7 $n=6$ ), and primed, challenged, and diphtheria toxin (DTX)-treated (lymph node D4 $n=8 ;$ D7 $n=4$; D10 $n=7$; lung D7 $n=6$ ) CD11bdiphtheria toxin receptor (DTR) mice were restimulated with phorbol 12-myristate 13-acetate (PMA) plus ionomycin and stained to compare cytokineproducing capabilities of $\mathrm{CD} 4^{+} \mathrm{T}$ lymphocytes. (c) Total numbers of cytokine-producing inflammatory $\mathrm{CD} 4{ }^{+} \mathrm{T}$ lymphocytes in the lungs, and (d) magnitude of cytokine production per cell was measured by the mean fluorescence intensity (MFI) of cytokine staining. Little differences in effector CD4 ${ }^{+}$ T lymphocytes were observed in lung-draining lymph nodes. In contrast, DTX treatment reduced the frequency, number, and intensity of interleukin (IL)13-, IL-4-, and IL-17A-producing CD4 ${ }^{+}$effector T lymphocytes in the lungs. Data are presented as medians. Statistical significance was calculated using Mann-Whitney $U$-test. ${ }^{*} P<0.05,{ }^{* \star} P<0.01$. Results represent two independent experiments. $\mathrm{D}$, day.

occurred outside the lungs in DTX-treated CD11b-DTR mice (Figure 5a and Supplementary Figures S4 and S5), and the numbers of $\mathrm{CD}_{11 \mathrm{~b}}{ }^{+}$DCs in the lung did not immediately change when half the macrophages were depleted (Figure 1b). Nonetheless, it is both plausible and of obvious importance that $\mathrm{CD}_{11 \mathrm{~b}}{ }^{+} \mathrm{DCs}$ might also be partially susceptible to depletion in our experiments, and the cumulative effects of DTX treatment include a delayed drop in the number of DCs present in lung and draining lymph node (Figure 4 and Supplementary Figure S6). Therefore, we compared our findings with the effect of targeting DCs for depletion using the CD11c-DTR system. Unlike CD11b-DTR mice, CD11c-DTR mice die 2-3 days after DTX injection because of toxic neurological effects on transgene-expressing nonhematopoietic cells. ${ }^{27}$ In order to study fibrosis, we generated bone marrow chimeras in which wild-type recipient mice received wild-type control, CD11b-DTR, CD11c-DTR, or CD11b/c-DTR double-transgenic donor bone marrow. Egg-sensitized chimeric mice were challenged IV with live $S$. mansoni eggs and then treated with DTX on days 3, 4, and 5 to deplete different leukocyte subsets and harvested at D7 (Figure 6a). Besides DCs, many resident airway and interstitial macrophages are $\mathrm{CD} 11 \mathrm{c}^{+}$, express the CD11c-DTR transgene, and can be depleted by DTX. ${ }^{28}$ However, despite this combinatorial depletion of cell populations, DTX treatment did not reduce granuloma volume or collagen deposition in CD11c-DTR chimeras (Figure 6b-d). In contrast, DTX-treated CD11b-DTR chimeras developed similarly reduced granuloma volume and fibrosis as nonchimeric 
a
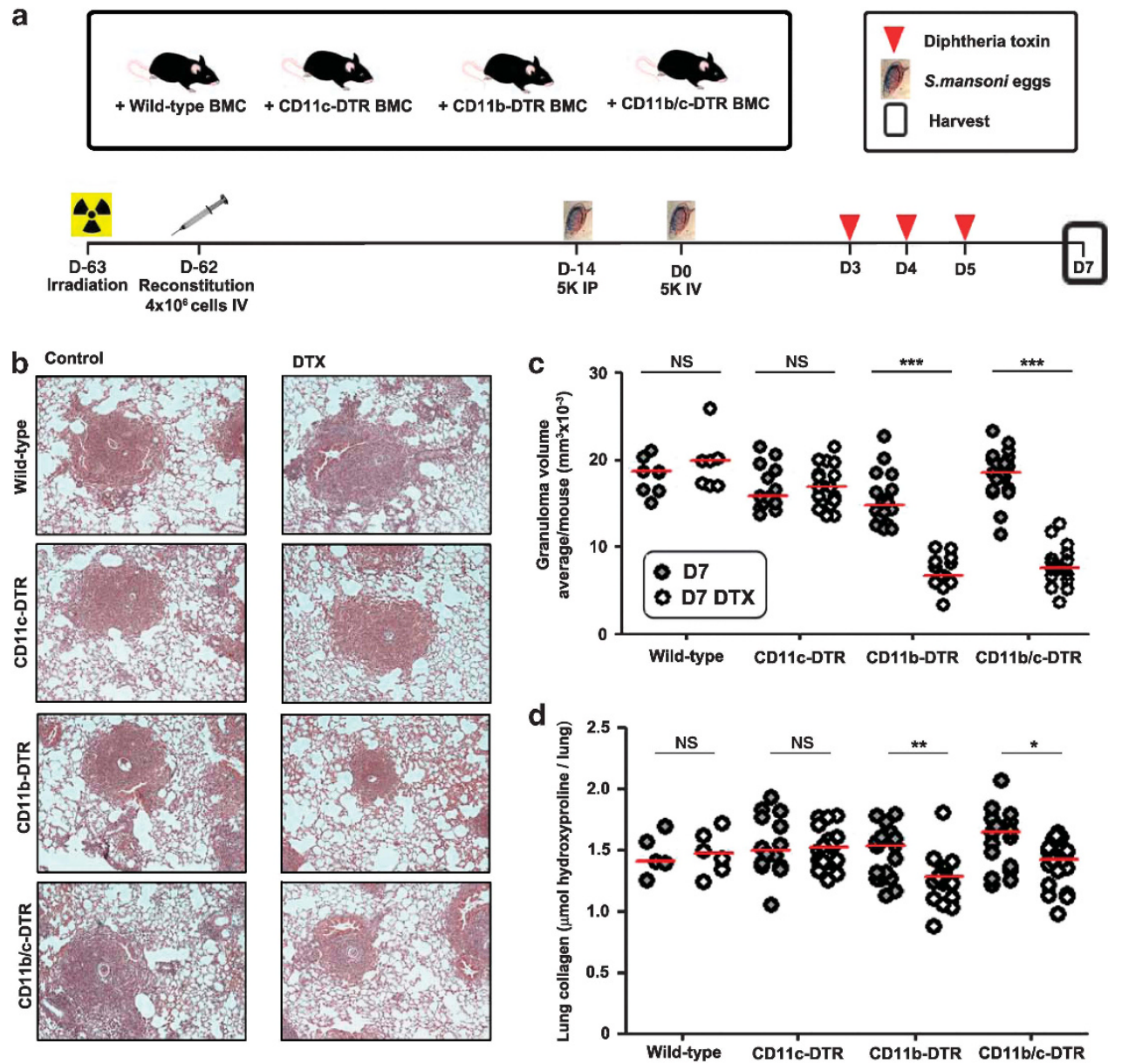

Figure 6 CD11c-diphtheria toxin receptor (DTR)-sensitive leukocytes are not required after antigen priming to maintain type 2-dependent granulomas and fibrosis in the lung. (a) Wild-type C57BI/6 mice were irradiated to ablate hematopoiesis and reconstituted with bone marrow cells (BMCs) from wildtype C57BI/6, CD11b-DTR, CD11c-DTR, or mice expressing both CD11b-DTR and CD11c-DTR transgenes (CD11b/c-DTR). Chimeric mice were primed and challenged with $S$. mansonieggs, and half were treated with diphtheria toxin (DTX;25 $\mathrm{ng} \mathrm{g}^{-1}$ ) when indicated. All mice were harvested at day 7 (D7). (b) Representative images of granulomas and collagen (blue) in lungs stained with Masson's trichrome (wild-type control $n=8$; wild-type DTX $n=7$; Cd11c-DTR control $n=14$; Cd11c-DTR DTX $n=18$; Cd11b-DTR control $n=18$; Cd11b-DTR DTX $n=14$; Cd11b/c-DTR control $n=19$; Cd11cDTR DTX $n=18$ ). (c) Lung granuloma volume was scored by a pathologist blinded to groups and data presented as average granuloma volume per mouse. (d) Lung collagen was measured by hydroxyproline content of all primed and egg challenged groups. DTX treatment of CD11b-DTR mice reduced both secondary lung granuloma volumes and collagen content of the lung. In contrast, DTX treatment of CD11c-DTR or CD11b/c-DTR mice did not change granuloma volumes or collagen content of the lung compared with DTX-treated wild-type or CD11b-DTR mice, respectively. Data are presented as median and statistical significance calculated using Mann-Whitney $U$-test. ${ }^{*} P<0.05,{ }^{\star \star} P<0.01,{ }^{\star * \star} P<0.001$. Results represent two independent experiments. IP, intraperitoneal; IV, intravenous; NS, not significant.

CD11b-DTR mice. Moreover, the CD11b/c-DTR doubly depleted chimeras showed no additional decrease in granuloma volume or fibrosis compared with CD11b-DTR chimeras. These data demonstrate that bone marrow-derived CD11bDTR-sensitive but not CD11c-DTR-sensitive leukocytes play a crucial role after antigen priming in maintaining Th2dependent granuloma formation and fibrosis in the lungs of $S$. mansoni egg-challenged mice.

We therefore compared which lung macrophage subsets were selectively depleted in CD11b-vs. CD11c-DTR transgenic mice by a single dose of DTX administered between days 3 and 4 after egg challenge. DTX-treated CD11c-DTR mice lost $\sim 80 \%$ of both $\mathrm{CD}_{11 \mathrm{~b}^{-}}$and $\mathrm{CD} 11 \mathrm{~b}^{+}$DCs in the granulomatous lung, but the total number of $\mathrm{CD}_{11 \mathrm{~b}^{+}} \mathrm{F} 4 / 80^{+}$ macrophages did not decrease (Figure 7a). However, Ly6C expression by the surviving macrophages differed dramatically, with the 1:1 ratio of Ly6C $\mathrm{C}^{+}$to $\mathrm{Ly} 6 \mathrm{C}^{-}$macrophages present in non-DTX-treated mice switching to 1:3 in CD11b-DTR mice and 10:1 in CD11c-DTR mice following DTX treatment (Figure 7b,c). In CD11b-DTR mice, DTX did not significantly alter the proportion of macrophages expressing MHCII or CD64 and only slightly increased the fraction expressing CD11c in the surviving population; only the loss of $\mathrm{Ly} 6 \mathrm{C}^{+}$cells was pronounced (Figure 7b, not shown). In contrast, in DTXtreated CD11c-DTR mice, the percentage of $\mathrm{CD} 11 \mathrm{c}^{+}$macrophages decreased in both the Ly6C-positive and -negative subsets, but the number of $\mathrm{Ly} 6 \mathrm{C}^{+}$macrophages actually increased, whereas the $\mathrm{Ly} 6 \mathrm{C}^{-}$population was almost entirely lost. Thus, the only macrophage subset whose depletion selectively correlates with the failure to maintain lung inflammation and fibrosis is the $\mathrm{Ly} 6 \mathrm{C}^{+}$population, most likely comprising recently recruited monocytes. 
a (i)

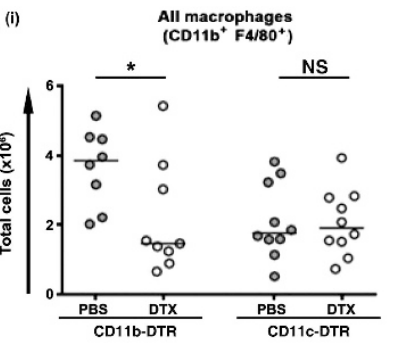

(ii)

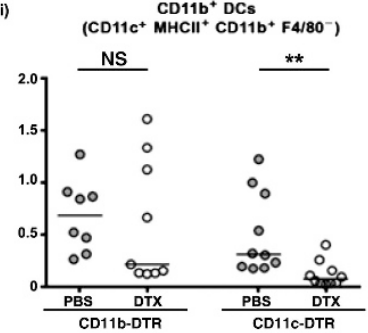

(iii)

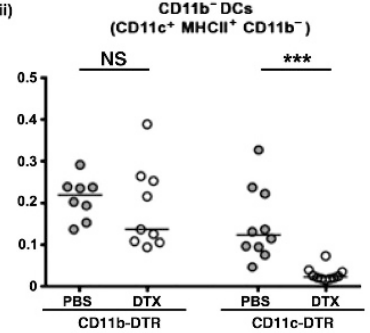

b

Gated on all macrophages $\left(\mathrm{CD}_{11 \mathrm{~b}}{ }^{+} \mathrm{F} 4 / 80^{+}\right)$

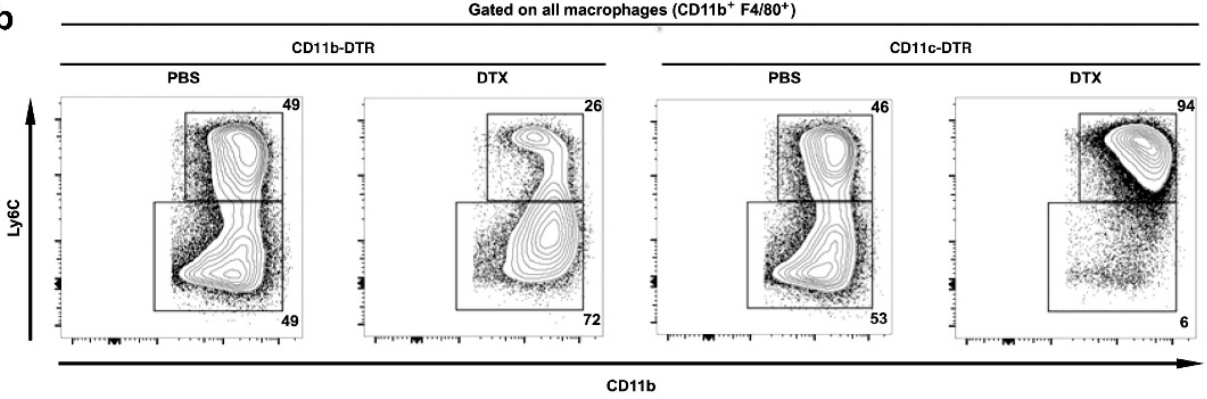

Gated on Ly6C+ macrophages (CD11 $\mathrm{b}^{+} \mathrm{F} 4 / 8 \mathrm{O}^{+} \mathrm{Ly}_{6 \mathrm{C}^{+}}$)
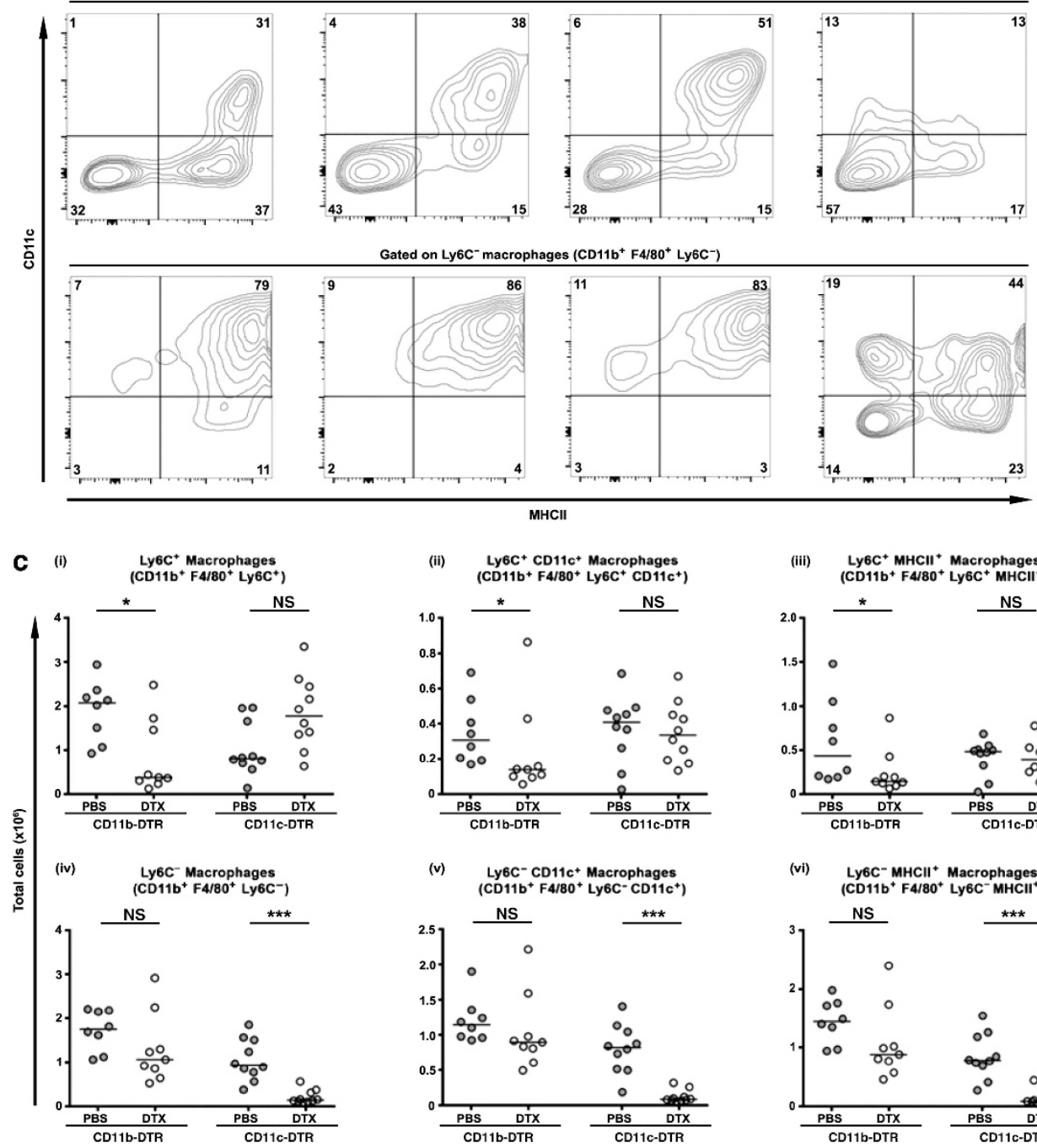

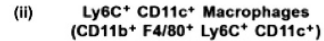

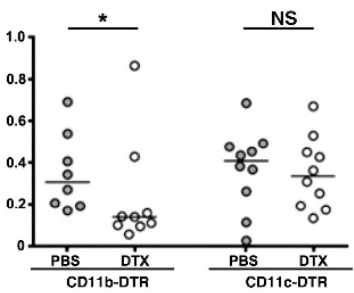

(v)

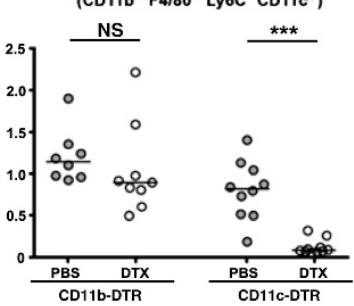

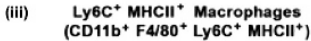

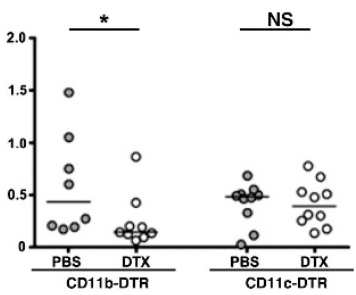

(vi) Ly6C- $\mathrm{MHCI}^{+}$Macrophages

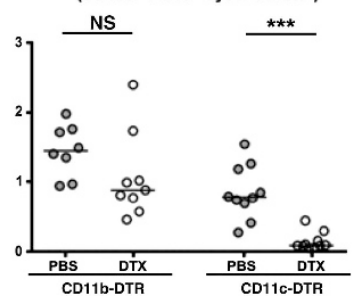


Macrophages regulate chemokine production and recruit effector $T$ cells to the lungs

We also examined whether macrophage depletion impaired effector T-cell homing to inflamed lungs using an adoptive transfer model. To do this we repeated the day 7 end point S. mansoni egg-induced lung granuloma model (Figure 2a), but also preactivated OT-II transgenic $\mathrm{CD} 4^{+} \mathrm{T}$ cells using soluble egg antigen to promote Th2 differentiation, delivered carboxyfluorescein succinimidyl ester (CFSE)-labeled effector T cells by IV injection on day 6 , and killed the animals $24 \mathrm{~h}$ later. As expected, very few but equal numbers of donor cells were recovered from the blood and uninvolved inguinal lymph nodes of control and depleted mice. In contrast, fewer CFSElabeled $\mathrm{T}$ cells homed to the lungs and lung-draining lymph nodes in the DTX-treated mice, suggesting macrophages were important for recruiting $\mathrm{T}$ cells to granulomatous tissues and/or their retention (Figure 8a).

We therefore screened lung tissue for changes in the expression of chemokines involved in the formation and regulation of S. mansoni egg-induced granulomas. ${ }^{29,30}$ We identified two chemokines, CCL1 and CCL22, whose diminished expression at both the mRNA and protein levels following depletion might impair Th2 effector cell recruitment (Figure 8b,c). CCL1 and CCL22 can be produced by IL-4/13stimulated macrophages, attract $\mathrm{CD}_{4}{ }^{+} \mathrm{T}$ cells, and their neutralization can reduce Th2-mediated lung inflammation. ${ }^{31,32}$ However, transcription of chemokines did not globally decrease. CCL2, a known monocyte chemoattractant, was increased at all time points following macrophage depletion, likely explaining why the $\mathrm{CD} 11 \mathrm{~b}^{+} \mathrm{F} 4 / 80^{+}$population in the lung shifted toward a monocyte phenotype after the last dose of DTX. In addition, DTX treatment did not reduce the eosinophil composition of granulomas (Figure $\mathbf{1 b}$, not shown), and consistent with this we observed little to no change in CCL11, a key eosinophil-attracting chemokine. Together, these data demonstrate that macrophages sustain effector $\mathrm{CD} 4{ }^{+} \mathrm{Th} 2$ responses in the lungs, at least in part, by producing $\mathrm{CD} 4{ }^{+} \mathrm{Th} 2$ cell-recruiting chemokines.

\section{CD11b-DTR-sensitive cells remain critical after systemic immunization to drive local type 2 immunity induced by airway allergen}

We next investigated whether established type 2 immunity to an airway allergen was similarly reduced by depleting CD11bDTR-sensitive cells. We employed a commonly used model of HDM-induced allergic lung inflammation and treated sensitized mice with DTX just before and during secondary airway challenge (Figure 9a). Depletion substantially impaired mucus production by airway epithelial cells (Figure $\mathbf{9 b}, \mathbf{d}$ ) and reduced the recruitment of leukocytes, particularly eosinophils, into the lung tissue and airway (Figure 9e). Ifng, Il13, and Il5 mRNA expression were also reduced in depleted mice as were IL-13-responsive genes including Clca3, Muc5ac, Arg1, Retnla, and Chi3l3 (Figure 9c).

In contrast, depletion had little to no effect on helper $\mathrm{T}$ cells in the lung-draining mediastinal lymph node, with the frequency of cytokine-producing $\mathrm{CD} 4^{+} \mathrm{T}$ cells either remaining unchanged (IL-4, IL-13, IL-17A) or increasing slightly $($ IFN- $\gamma$ ) (Supplementary Figure S7a). In the lungs, the per-cell cytokine response of $\mathrm{CD} 4{ }^{+} \mathrm{T}$ cells was also unaffected (not shown); however, their total numbers were markedly reduced (Supplementary Figure S7b,c). Together with the results of related studies, ${ }^{16,33}$ our data emphasize that homing of Th2 effector cells into lung can depend critically on signals from monocyte-derived cells recruited in the same time frame.

\section{Macrophage depletion during Nippostrongylus infection disrupts type 2 cytokine production and suppresses multiple host defense mechanisms in the lung and intestine}

Type 2 immunity can cause pathologies but its natural benefit is to protect against infections by pathogens such as parasitic hookworms. N. brasiliensis larvae infect mice through the skin, traverse the lungs, and ultimately reach the small intestine where they lay eggs before being expelled in $8-12$ days by the host's Th2 response enhancing smooth muscle contractility and mucus production. ${ }^{12,34}$ In addition, the type 2 response ameliorates the damage caused by parasites migrating through the lungs. ${ }^{10}$ We revisited the question of how macrophages contribute to these processes by treating infected CD11b-DTR mice with DTX as parasites migrate through the lungs (day 4), before peak egg production (day 7), and during expulsion (day 10) (Figure 10a). Macrophage depletion did not affect $N$. brasiliensis from maturing and reaching the intestine by day 7 , but did impair expulsion of the parasite at day 10 and increased fecundity throughout the infection (Figure 10b-e).

Delayed parasite expulsion was not due to skewing toward a type 1 or suppressive immune response as Ifng transcript levels did not change and Il10 was reduced at day 7 following depletion (Figure 10f). Instead, targeting macrophages substantially impaired the type 2 response in the gut. Although Th2-stimulated mucus genes were only

Figure 7 Failure to maintain lung inflammation and fibrosis selectively correlates with the depletion of Ly6C ${ }^{+}$lung macrophages in CD11b-diphtheria toxin receptor (DTR) mice. CD11b-DTR and CD11c-DTR egg-primed and -challenged transgenic mice were treated with diphtheria toxin (DTX) or phosphate-buffered saline (PBS) on day 3 (D3) and harvested on day 4 (D4). Lung leukocytes were analyzed by flow cytometry to identify directly depleted cell types (CD11b-DTR PBS $n=8$, CD11b-DTR DTX $n=9$, CD11c-DTR PBS $n=10$, CD11c-DTR DTX $n=10)$. (a) Numbers of dendritic cells and total macrophages in the lung, based on gates shown in Figure 1a. (b) Reciprocal depletion of the Ly6C ${ }^{+}$macrophage subset in CD11b-DTR and Ly6C $^{-} \mathrm{CD}_{11 \mathrm{C}}{ }^{+}$subset in CD11C-DTR mice, and profiles of CD11c and MHCll coexpression by these subsets. Percentages of gated cells from individual mice are representative of group means. (c) DTX treatment decreased the number of Ly6C ${ }^{+}$macrophages in CD11b-DTR but not CD11c-DTR mice, whereas Ly6 $\mathrm{C}^{-}$macrophages decreased in CD11c-DTR but not CD11b-DTR mice. Data are presented in a and $\mathbf{c}$ as median. Statistical significance was calculated using Mann-Whitney U-test. ${ }^{\star} P<0.05$, ${ }^{\star \star} P<0.01,{ }^{\star \star \star} P<0.001$. Results represent two independent experiments. NS, not significant. 
marginally reduced (Clca3) or unaffected $(M u c 5 a c)$, we found impaired expression of Retnla, Retnlb, and Chi3l3. In the lung, erythrocytes released into the airways by parasite-induced hemorrhage were inefficiently cleared following depletion (not shown) and Clca3 or Muc5ac failed to increase (Figure 10g). Our findings match similar studies, ${ }^{10,12}$ but we additionally observed that depleting macrophages prevented or reduced the induction of Il5 and Il13 in both the intestine and lung (Figure 10f,g). Again, this was a localized defect in type 2 immunity because depletion caused scant decreases in cytokine production by $\mathrm{CD} 4{ }^{+} \mathrm{Th} 2$ cells in the mesenteric lymph nodes (Supplementary Figure S8), a

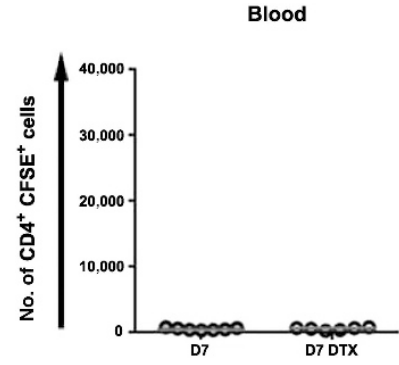

Inguinal LN

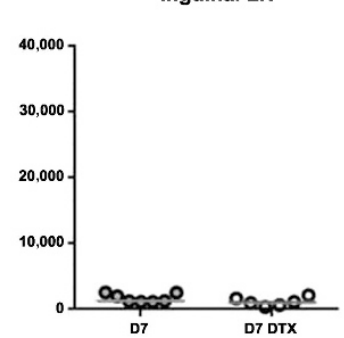

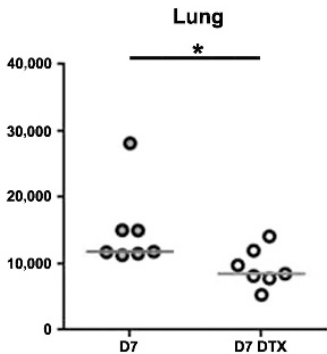
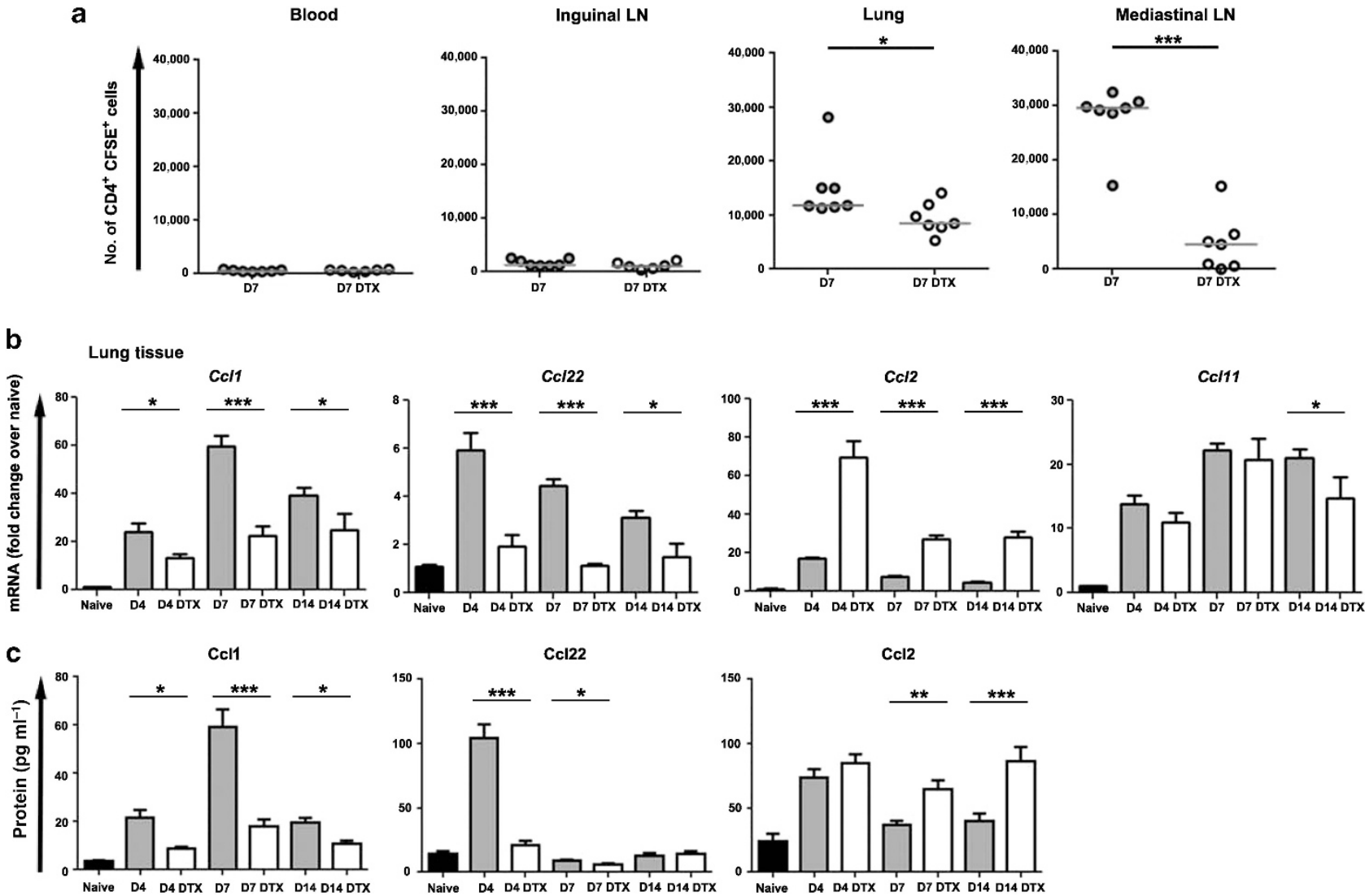

Figure 8 Macrophages regulate chemokine production and recruit effector T cells to the lungs. (a) In vitro activated carboxyfluorescein succinimidyl ester (CFSE)-labeled OT-II transgenic CD4 ${ }^{+}$T lymphocytes $\left(4 \times 10^{6}\right.$ cells) were injected intravenously (IV) into egg-primed and -challenged CD11bdiphtheria toxin receptor (DTR) mice with or without prior diphtheria toxin (DTX) treatment on day 6 (D6). The homing of donor T lymphocytes was compared $24 \mathrm{~h}$ after transfer by detecting $\mathrm{CFSE}^{+} \mathrm{CD}^{+}{ }^{+} \mathrm{T}$ cells in different tissues by flow cytometry. Fewer activated CD4 ${ }^{+} \mathrm{T}_{\text {lymphocytes }}$ were recruited to and remained in the lung and mediastinal lymph nodes (LNs) in DTX-treated mice. In contrast, similar small numbers of donor cells were present in blood or inguinal LNs. Relative quantitative gene (b) and protein expression (c) of chemokines in lung tissue from naive ( $n=13$ ), egg primed and challenged (D4 $n=8 ; \mathrm{D} 7 n=9$, D10 $n=10$ ), and primed, challenged, and DTX-treated (D4 $n=8 ; \mathrm{D} 7 n=10$, D10 $n=9$ ) CD11b-DTR mice. Results were normalized to RPLP2 and scaled to naive mice. Induction of CCL1 and CCL22 was blunted by DTX treatment, whereas CCL2 was further increased at all time points and CCL11 remained mostly unaltered. The data in a are presented as median. The data in $\mathbf{b}$ and $\mathbf{c}$ are presented as mean \pm s.e.m. Statistical significance was calculated using unpaired two-tailed Student's $t$-test or Mann-Whitney U-test as appropriate. ${ }^{*} P<0.05$, ${ }^{* \star} P<0.01$,

${ }^{* \star *} P<0.001$. Results represent two independent experiments.

Figure 9 CD11b-diphtheria toxin receptor (DTR)-sensitive cells locally drive airway allergen-induced type 2 immunity. (a) CD11b-DTR mice were primed by intraperitoneal (IP) injection of house dust mite (HDM, $200 \mu \mathrm{g}$ ) on day 0 (D0) and day 7 (D7) before intratracheal (IT) challenge with HDM (50 $\mu \mathrm{g})$ on D14 and D16. Half of the mice were treated with diphtheria toxin (DTX, $25 \mathrm{ng} \mathrm{g}^{-1}$ ) at D13 and D15. Lungs and lung-draining mediastinal lymph nodes were harvested on D17. (b) Representative images of airway epithelial mucus by Alcian blue/periodic acid-Schiff (AB-PAS) staining of saline-treated (naive $n=11$ ), HDM primed and challenged (HDM $n=12$ ), and primed, challenged, and DTX-treated (HDM DTX $n=10)$ CD11b-DTR mice. (c) Relative quantitative gene expression in lung tissue was normalized to RPLP2 and scaled to naive mice. DTX treatment weakened the HDM-stimulated increase in mucus and Thelper type 2 (Th2)-induced genes. (d) AB-PAS staining (scored 0-4 by a pathologist blinded to groups) was less intense and widespread in the airways of DTX-treated mice. (e) Total number of leukocytes in perfused lung tissue and bronchoalveolar lavage (BAL). Total number of eosinophils in BAL was calculated from cytospin analysis. DTX treatment decreased the total number of leukocytes in lung tissue and BAL, as well as airway eosinophils. Data are presented as mean \pm s.e.m. Statistical significance was calculated using unpaired two-tailed Student's $t$-test. ${ }^{\star} P<0.05$, ${ }^{* \star} P<0.01$, ${ }^{* * \star} P<0.001$. Results represent two independent experiments. 
a

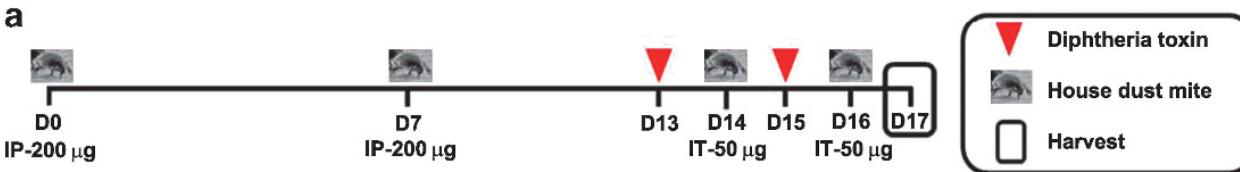

b

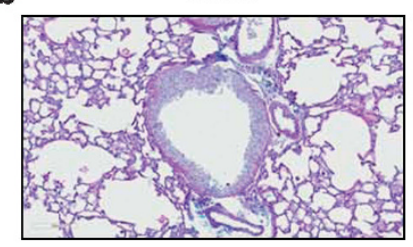

c
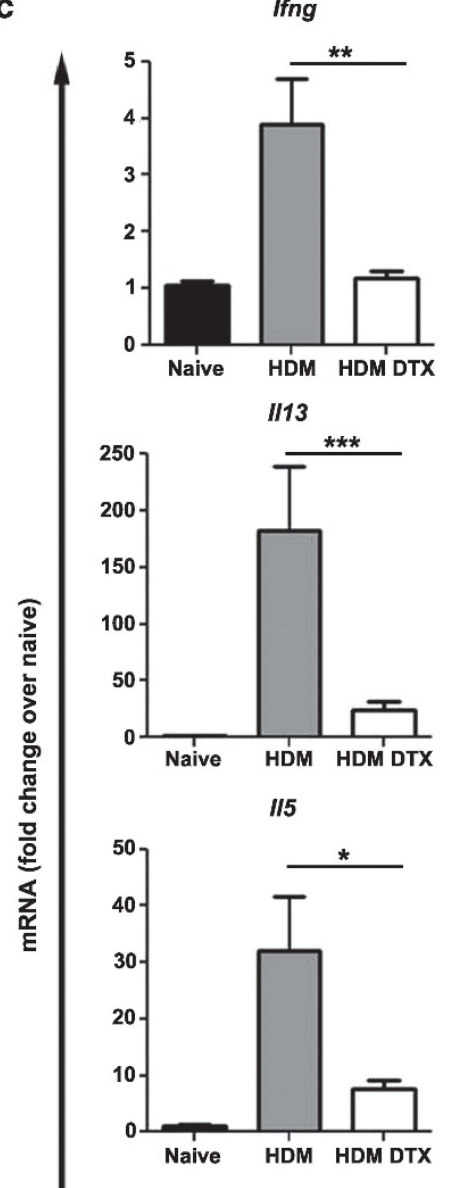

Clca3

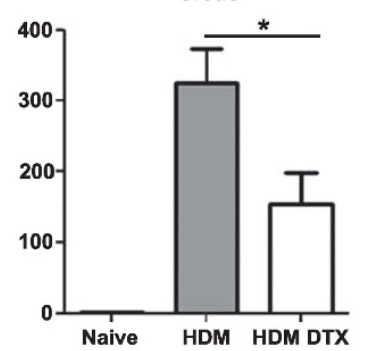

HDM

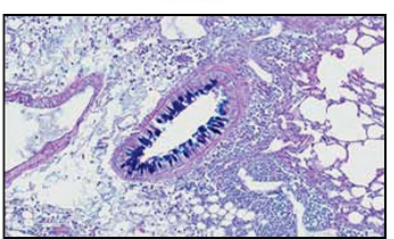

Muc5ac
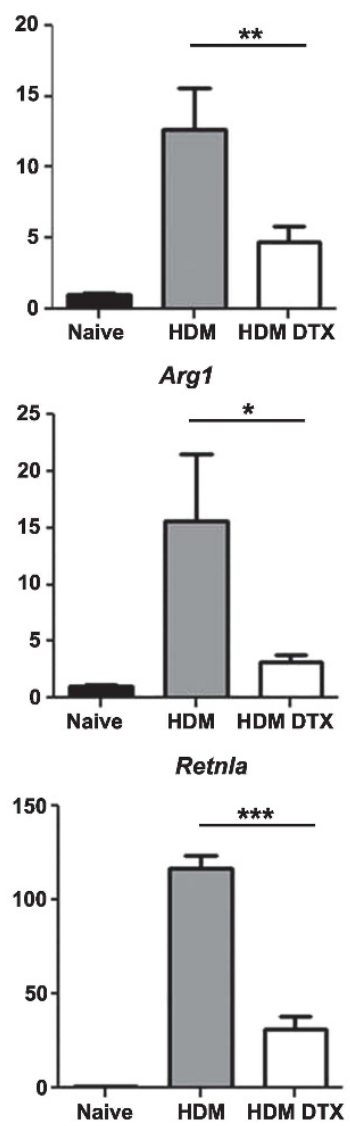

Chi3/3

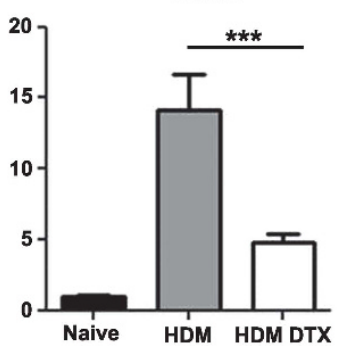

HDM DTX

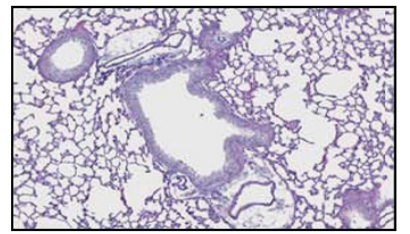

d Epithelial mucus

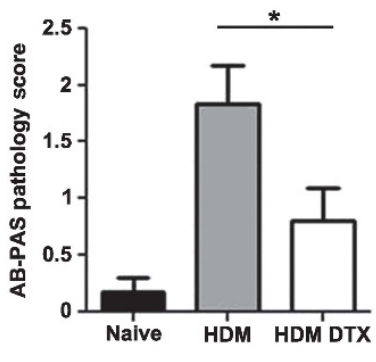

e Lung leukocytes

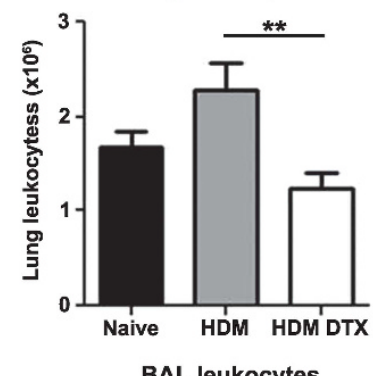

BAL leukocytes

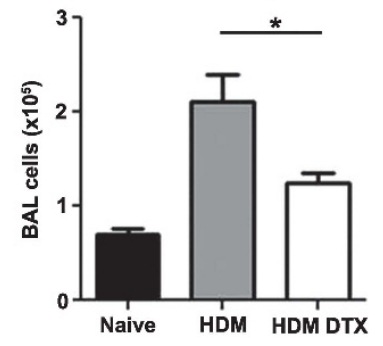

BAL eosinophils

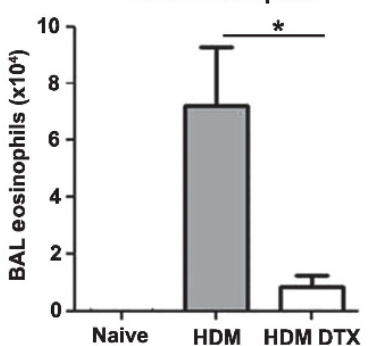


suggesting that effector $\mathrm{T}$ cells rely on macrophages to guide them into inflamed tissue.

Thus, in three distinct models our results argue that $\mathrm{CD} 11 \mathrm{~b}^{+}$ $\mathrm{F} 4 / 80^{+}$macrophages play an indispensable role in maintaining type 2 immune responses in the lung and gut and that even partially interfering with this function reduces inflammation, fibrosis, and host defense mechanisms by disrupting the recruitment of IL-13-producing $\mathrm{CD} 4^{+} \mathrm{T}$ cells.

\section{DISCUSSION}

Macrophages are phagocytic cells that detect, engulf, and destroy microbes; yet, this function is only one of the many important roles they play in the immune response. In addition to aiding in host defense, macrophages regulate tissue development, help maintain and restore organ function, regulate metabolic pathways, dispose of dying cells and cellular debris, and promote or resolve inflammation. ${ }^{3,9,35-39}$ One reason macrophages are capable of playing such diverse roles is that their activation state is not fixed, enabling them to adapt and alter their function in response to changes in the local environment. This inherent plasticity is readily apparent in tissue repair and fibrosis, where macrophages are thought to play both pro- and anti-fibrotic roles at various stages of wound healing responses. ${ }^{37,39,40}$

Here we present evidence that macrophages must continue to recruit, and perhaps retain, effector CD4 T cells in the lung or type 2 immune responses will begin to abate. We believe this is a novel and important discovery because it demonstrates mechanistically how even strong and fully developed IL-13dependent fibrosis and other type 2-driven inflammatory pathologies could be disrupted by transiently targeting one type of leukocyte. In this study, using three distinct Th2 models and CD11b-DTR and CD11c-DTR transgenic mice, we showed that $\mathrm{CD}_{11 \mathrm{~b}^{+}} \mathrm{F} 4 / 80^{+}$macrophages but not $\mathrm{CD} 11 \mathrm{c}^{+} \mathrm{DCs}$ are critical to the maintenance of type 2-driven tissue inflammation and lung fibrosis but are less critical to the maintenance of type 2 immunity in secondary lymphoid organs. The S. mansoni egg-induced granuloma model creates an extremely robust type 2 inflammatory response that develops synchronously and in distinct stages in the lung, with $>50 \%$ of all $\mathrm{CD} 4{ }^{+} \mathrm{T}$ cells infiltrating the lung capable of producing IL-13. In antigenprimed mice, this model of IL-13-dependent fibrosis is robust enough to more than double the collagen content of the lung. We designed our experiments to compare the consequences of depleting macrophages during the formative, maintenance, and resolution stages of IL-13-driven inflammation and fibrosis. Surprisingly, even when macrophages were depleted after granulomas had formed and fibrosis was established, we found a rapid, large, and broad reduction in the type 2 immune response in the lung but not in the draining lymph node, with no evidence that macrophages were switching their phenotype in aggregate from inducers to resolvers of inflammation and fibrosis. These results demonstrate that macrophages are required to maintain IL-13dependent fibrogenesis in the lung even during the stage when inflammation is clearing.
Previous studies have examined the roles of different leukocyte populations at the onset of immune activation and lung pathology using the CD11b-DTR, CD11c-DTR, and clodronateloaded liposome depletion technologies, as well as in CCR2deficient mice to impair monocyte mobilization..$^{10,16-18,28,33,41}$ All such data, including the results we present here, warrant cautious interpretation as macrophage depletion by any of these methods is incomplete and varies by location, duration, and cellular subset. Overall, these studies concluded that monocyte-derived populations play critical roles at the start of inflammatory responses. However, these tissue-infiltrating monocytes exhibited phenotypes and functions that made it difficult to neatly categorize them as "macrophages," "monocytes," or "dendritic cells." The CD11c-DTR and clodronate methods have been reported to target a wider range of cell populations in inflamed lungs than CD11b-DTR mice. In our studies, DTX treatment depleted approximately half of all the CD11b-DTR F4 $/ 80^{+}$macrophages in the inflamed lung, and primarily the $\mathrm{Ly} 6 \mathrm{C}^{+}$subset, without ablating eosinophils, neutrophils, or $\mathrm{F} 4 / 80^{-}$dendritic cells. In our experimental system, we found DTX-treated CD11c-DTR mice lost CD11bDCs, CD11b ${ }^{+}$DCs, and CD11c ${ }^{+}$Ly6C-but not Ly6C $^{+}$ macrophages. Our results support investigations of HDMinduced lung inflammation that showed that chemokine secretion and antigen presenting by "monocyte-derived dendritic cells" in the lung are crucial to the initiation of type 2 lung inflammation. ${ }^{33,42}$ In our study, we identified that a CD11bDTR-sensitive $\mathrm{F} 4 / 80^{+} \mathrm{Ly} 6 \mathrm{C}^{+}$lung macrophage population, likely similarly derived from monocytes, is critical to maintain inflammatory type 2 immune responses. We did not design this study to discriminate between macrophages of different origins. However, the number of macrophages, disproportionate depletion of the $\mathrm{Ly}_{6 \mathrm{C}}{ }^{+}$subset, and pattern of CCL2 expression most likely indicate large-scale, CD11b-DTR-sensitive monocyte immigration into the lungs, as was recently described in the liver of $S$. mansoni-infected mice. ${ }^{43}$ In contrast, the $\mathrm{CD} 11 \mathrm{~b}$ (low) $\mathrm{CD} 11 \mathrm{c}^{+}$alveolar subset of resident macrophages is reported to resist depletion during challenge in CD11b-DTR mice, ${ }^{16-18}$ suggesting that lung inflammation and fibrosis in our experiments crucially depends on monocyte-derived macrophages.

Although macrophages were critical to the maintenance of established type 2 responses in the affected tissues, there was no obvious diminution in the differentiation and activation of $\mathrm{CD} 4{ }^{+}$Th2 cells in the draining lymph nodes. Such compartmentalization adds to the preponderance of evidence that DCs, but not monocyte-derived or resident macrophages, initiate $\mathrm{T}$-cell responses in lymph nodes. In some cases monocytes also function and/or differentiate into DCs, ${ }^{33,44}$ but such a role is dispensable outside the lung and after antigen priming in our experiments. Conversely, CD11c-DTR-dependent depletion beginning 3 days after challenging antigen-primed mice did not reduce inflammation or fibrosis in the lung, and combining CD11c-DTR depletion had no additional impact over CD11b-DTR alone in bone marrow chimeras. We also found CD11c-DTR transgenic mice lost $\sim 80 \%$ of both $\mathrm{CD}_{11 b^{-}}$and 
a

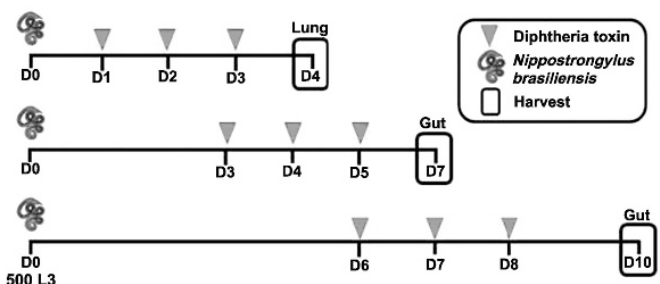

c

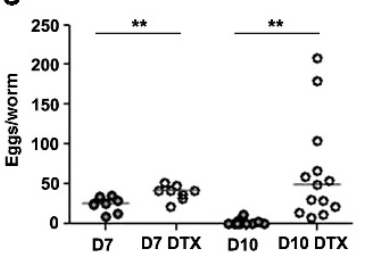

d

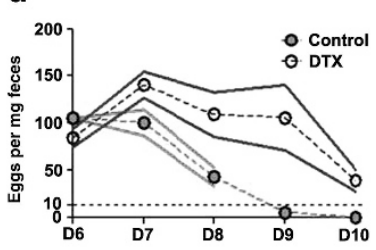

b

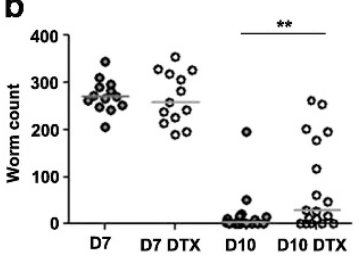

e

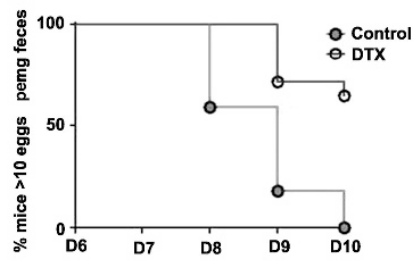

f Gut

Ifng
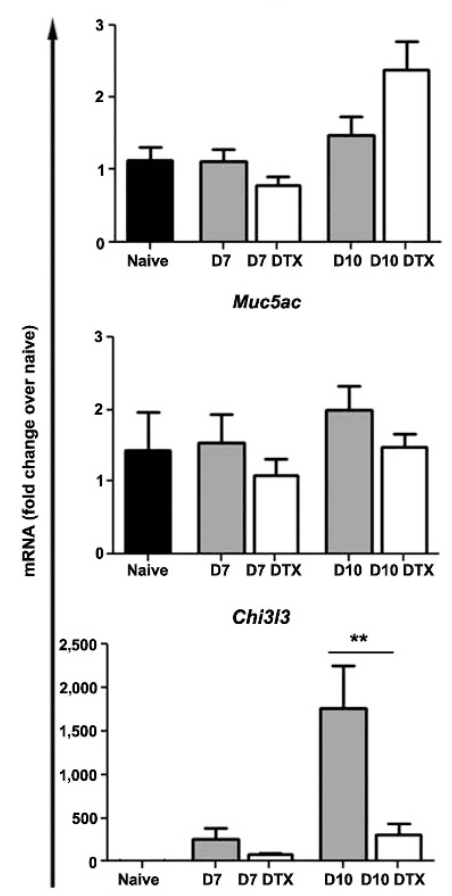
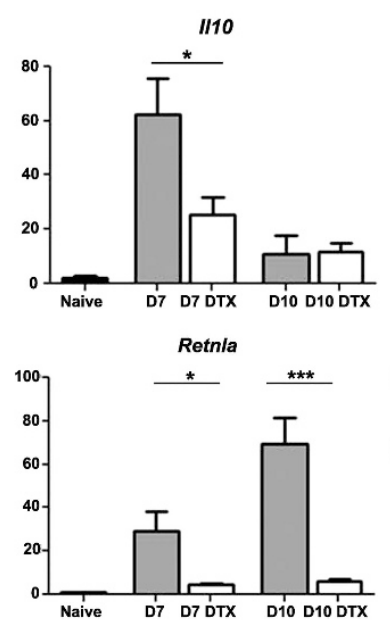

II5

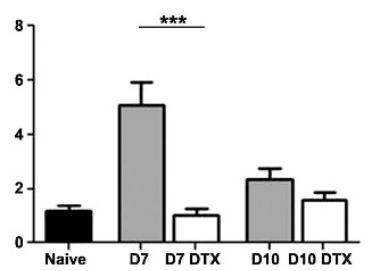

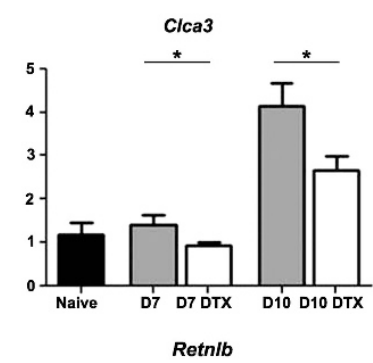

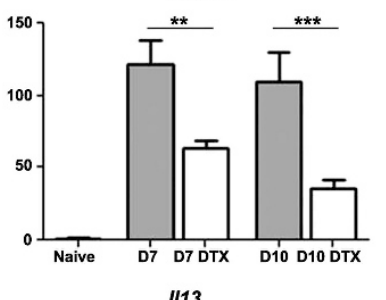

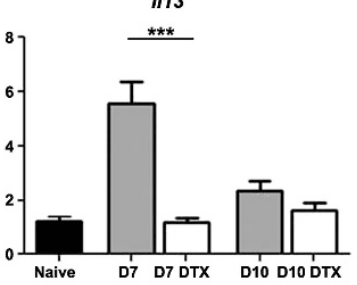
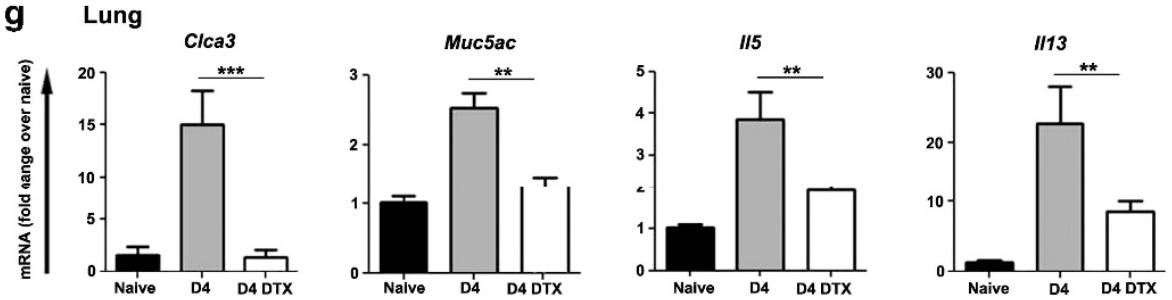

Figure 10 Macrophage depletion suppresses T helper type 2 (Th2) gene expression and impairs defense against Nippostrongylus brasiliensis hookworm infection. (a) CD11b-diphtheria toxin receptor (DTR) mice were injected subcutaneously with N. brasiliensis (500 L3) at day 0 (D0). Mice were harvested at D4 (lung), or D7 or D10 (gut). Half of the mice were treated with diphtheria toxin (DTX, $25 \mathrm{ng} \mathrm{g}^{-1}$ ) when indicated. (b) Intestinal worm burden was equal in control mice and DTX-treated mice on D7 ( $n=13 /$ group) but significantly higher in DTX mice at D10 ( $n=18 /$ group). (c) DTX treatment increased fecundity of $N$. brasiliensis adults (eggs/worm) at both D7 and D10. Daily longitudinal fecal egg counts showed (d) a higher egg burden (eggs per mg feces) from D7 ( $n=8 /$ group) to D10 (control $n=10$; DTX $n=14$ ) and (e) an increased percentage of infected mice above a threshold (dotted line in e) of $>10$ eggs per mg feces following DTX treatment (control $n=22$; DTX $n=14$ ). Relative quantitative gene expression in (f) D7 and D10 gut (grossly inflamed proximal duodenum) and (g) D4 lung tissue from untreated (naive $n=10$ ), N. brasiliensis-infected (D4 $n=8 ; \mathrm{D} 7 n=13 ; \mathrm{D} 10 n=18$ ), and infected DTX-treated (DTX) (D4 $n=9 ; \mathrm{D} 7 n=18 ; \mathrm{D} 10 n=28)$ CD11b-DTR mice. Results are normalized to RPLP2 and scaled to naive mice. The majority of Th2-dependent genes exhibited weaker induction following DTX treatment in infected mice. The data in $\mathbf{b}$ and $\mathbf{c}$ are presented as median. The data in $\mathbf{d}$, $\mathbf{f}$, and $\mathbf{g}$ are presented as mean \pm s.e.m. Statistical significance was calculated using unpaired two-tailed Student's $t$-test or Mann-Whitney $U$-test as appropriate. ${ }^{*} P<0.05,{ }^{* *} P<0.01,{ }^{* * *} P<0.001$. Results represent two independent experiments. 
$\mathrm{CD} 11 \mathrm{~b}^{+}$DCs in the granulomatous lung after 1 dose of DTX. Therefore, the DCs depleted in this system are not required after initiation to maintain established type 2 responses in the lung, further emphasizing the important localized role played by $\mathrm{CD} 11 \mathrm{~b}^{+} \mathrm{F} 4 / 80^{+} \mathrm{Ly} 6 \mathrm{C}^{+}$macrophages.

Why macrophages are critical to the maintenance of type 2 immunity was difficult to pin down because macrophages produce a variety of growth factors, cytokines, and chemokines that can diversely influence the maintenance of adaptive immune responses. As type 2 immunity was reduced in the tissues but not in the draining lymph nodes of depleted mice, we tested T-cell homing to the inflamed lungs. Strikingly, we showed that depleting macrophages reduced the number of preactivated and transferred Th2 cells in the lungs and lungdraining mediastinal lymph nodes. As effector $\mathrm{T}$ cells do not generally home directly to lymph nodes, the combined decrease of donor cells in both locations 1 day after transfer likely results from impaired recruitment from the bloodstream to the lungs followed by drainage from lung to lymph node. Our use of ovalbumin-specific $\mathrm{T}$ cells likely favors emigration from the lungs because these cells cannot locate their cognate antigen, and also adds evidence discriminating between weaker recruitment and a lower probability of locating and being retained in contact with an antigen-presenting cell. We therefore interpret our results to mean that macrophage depletion impaired the recruitment, and/or retention, of $\mathrm{CD} 4{ }^{+}$effector $\mathrm{T}$ cells to the lungs of $S$. mansoni egg-challenged mice.

Furthermore, chemokines CCL1 and CCL22, which are known $\mathrm{CD} 4{ }^{+}$Th2 cell chemoattractants produced by IL-4/13stimulated macrophages, were markedly decreased in the lungs following DTX treatment, providing at least one explanation for the decreased type 2 responses in the lung and gut. We also found correlating evidence that the reduced number of effector $\mathrm{T}$ cells still homing to the lungs after depletion were additionally impaired because of decreased antigen presentation. CD4 T cells recovered from the lungs, but not draining lymph nodes, of DTX-treated CD11b-DTR mice produced less cytokines both as a percent of the population and per cell. In parallel, DTX treatment shifted the predominant phenotype of macrophages on day 7 from $\mathrm{MHCII}^{+} \mathrm{CD}_{11 \mathrm{c}^{+}} \mathrm{Ly}_{6 \mathrm{C}}^{-}$to $\mathrm{MHCII}^{-}$ CD11c ${ }^{-}$Ly6C $^{+}$. This shift suggests that most of the monocytes would otherwise develop into functioning antigen-presenting cells and the depleted populations are replaced by cells yet to acquire the same functions. However, full-scale lung inflammation and fibrosis developed despite targeting $\mathrm{CD}_{11 c^{+}}$leukocytes.

In our experiments, Th17 cells outside the lungs are scarcely affected by macrophage depletion (Figure 5a and Supplementary Figures S5, S7a, S8). However, as for Th2 cells, loss of macrophages decreased the IL-17A ${ }^{+}$CD4 T-cell population within the lung primarily by reducing the total number of $\mathrm{T}$ cells (Figure 5b-d and Supplementary Figure S7c). Recruitment of both Th2 and Th17 cells to the granulomatous lungs may therefore depend on macrophages. However, DTX treatment increased neutrophil inflammation without causing lung hemorrhage, necrosis, and the characteristic pathologies of severely disabled Th2 and/or exacerbated Th17 responses. ${ }^{10,45,46}$ This suggests either another source of IL-17A increases or that neutrophils are recruited by an IL-17A-independent mechanism, but also that this influx of neutrophils does not damage the lung and perhaps aids in clearing extracellular matrix. A likely beneficial effect from neutrophils in IL-13-dependent fibrosis would contrast with their presumed role as a profibrotic effector in other models, such as IL-1- and transforming growth factor$\beta$-dependent bleomycin-induced lung injury. ${ }^{47}$

Previous studies using clodronate liposomes to deplete phagocytic cells suggested that alternatively activated macrophages, which predominate during infection with the nematode Heligmosomoides polygyrus, are directly involved in parasite expulsion from the gut. ${ }^{10}$ Although the exact antiparasite mechanism was not identified, the authors hypothesized that macrophages could directly impair parasite mobility and fitness as suggested in a study by Bieren et al. ${ }^{48}$ Surprisingly, clodronate treatment caused no reduction in IL-4 or IL-13 gene expression in the mesenteric lymph nodes or proximal small intestines, suggesting unimpaired T-cell activation and homing despite phagocyte depletion. In contrast, in our studies using CD11b-DTR mice we observed a marked reduction in type 2 tissue cytokine expression in all three Th2 models examined. In the case of $N$. brasiliensis infection, this resulted in a weaker induction of several mechanisms implicated in antinematode immunity, including mucin production and Retnlb expression. ${ }^{10,12}$ Thus, although macrophages also participate directly in anti-nematode immunity, our findings suggest that they are critically and more broadly involved in the maintenance of the entire type 2 host defense and tissue repair programs in the lung and gut.

In conclusion, our studies from three different Th2 models demonstrate that macrophages contribute to the maintenance of type 2 immune responses in the lung and gut. They may play this role after the initiation of an immune response by producing chemokines that recruit effector $\mathrm{T}$ cells to inflamed tissue and by presenting antigens to reactivate the arriving $\mathrm{T}$ cells. Importantly, this role for macrophages appears to be needed continuously by ongoing type 2 immune reactions in the lung and gut. Thus, therapies designed to transiently but specifically target the migration, survival, or activation of macrophages could emerge as viable treatments for a host of important diseases that are defined by the persistent overproduction of type 2-associated cytokines and chronic inflammation.

\section{METHODS}

Materials, mice, and macrophage depletion. All reagents were from Sigma Aldrich (St Louis, MO) unless stated otherwise. All experiments were performed at the National Institutes of Health (Bethesda, MD) using mice bred and housed under specific pathogen-free conditions in an American Association for the Accreditation of Laboratory Animal Care-approved facility under Animal Study Proposal LPD-16E. The National Institute of Allergy and Infectious Diseases (NIAID) animal care and use committee approved all experimental procedures.

CD11b-DTR (FVB-Tg(ITGAM-DTR/EGFP)34Lan/J) and CD11cDTR (B6.FVB-Tg(Itgax-DTR/EGFP)57Lan/J) transgenic mice were 
obtained from The Jackson Laboratory (Bar Harbor, ME). CD11cDTR mice were crossed with CD45.1 congenic mice (B6.SJL-Ptprc ${ }^{\mathrm{a}}$ $\mathrm{Pepc}^{\mathrm{b}} /$ BoyJ). Homozygous colonies were maintained on a $\mathrm{C} 57 \mathrm{Bl} / 6$ background and either crossed with wild-type mice (Taconic, Germantown, NY) to produce hemizygous transgenic mice for experiments or intercrossed to generate doubly hemizygous transgenic mice (CD11b/c-DTR).

DTX (Sigma Aldrich) was reconstituted to $2 \mathrm{mg} \mathrm{ml}^{-1}$ in sterile water and then diluted to $2.5 \mu \mathrm{g} \mathrm{ml}^{-1}$ in sterile saline. Mice were randomly assigned to control and experimental groups. Experimental mice were injected via the intraperitoneal (IP) route with DTX $\left(25 \mathrm{ng} \mathrm{g}^{-1}\right)$ as indicated for each experimental model.

Schistosome egg-induced lung granulomas. S. mansoni eggs were provided by the Biomedical Research Institute (Rockville, MD). For the secondary lung granuloma model, mice were primed with IP injection of 5,000 eggs in phosphate-buffered saline (PBS) on D-14 and injected IV with 5,000 live eggs in PBS at D0 to induce lung granulomas. Half of the mice were treated with DTX $\left(25 \mathrm{ngg}^{-1}\right)$ when indicated (Figure 2a). Mice were killed by pentobarbital overdose plus heparin on D4, D7, or D14. For the primary lung granuloma model, mice were treated as above without IP priming. For histological analyses, matched lung lobes were washed with PBS, inflated with Bouin's fixative, and stained with Masson's trichrome. Granuloma volume was determined by an experienced pathologist blinded to groups scoring 5-30 granulomas per mouse. For assessment of fibrosis, matched lung lobes were weighed and digested with $2 \mathrm{ml}$ of $6 \mathrm{~N} \mathrm{HCl}$ to measure the quantity of hydroxyproline, ${ }^{49}$ or sections were stained with picrosirius red to evaluate collagen distribution. Matched samples of lung tissue for quantitative PCR were collected and processed as below.

HDM treatment. Mice were primed by IP injection of $200 \mu \mathrm{g}$ of HDM (Greer, Lenoir, NC) in sterile saline on D0 and D7. Primed mice were challenged by intratracheal instillation of $50 \mu \mathrm{g}$ of HDM in $30 \mu \mathrm{l}$ of sterile saline (or $30 \mu \mathrm{l}$ of sterile saline as a control) on D14 and D16. Half the mice were treated with DTX $\left(25 \mathrm{ngg}^{-1}\right)$ at D13 and D15 (Figure 9a). Mice were killed by pentobarbital overdose plus heparin $24 \mathrm{~h}$ after the last HDM challenge and their tracheas cannulated with an Insyte venous catheter (BD, San Jose, CA). Bronchoalveolar lavage was performed with ice-cold PBS supplemented with 5 mm EDTA. Percentage of eosinophils was determined using cytospin preparations stained with Diff-Quick (Boehringer, Ridgefield, CT) by evaluating $>200$ cells per slide. For histological analyses of epithelial mucus, lung lobes were inflated with Bouin's fixative, stained with Alcian blue/ periodic acid-Schiff, and scored by an experienced pathologist blinded to groups. Matched samples of lung tissue for quantitative PCR were collected and processed as below.

Nippostrongylus infection. Preparation and the subcutaneous inoculation of mice with 500 infective third-stage $N$. brasiliensis larvae (L3) were performed as previously described. ${ }^{34} \mathrm{~N}$. brasiliensis eggs were initially provided by Dr Joe Urban (USDA, Beltsville, MD). Half the mice were treated with DTX $\left(25 \mathrm{ngg}^{-1}\right)$ when indicated (Figure 10a). Mice were killed by pentobarbital overdose plus heparin on D4 (for lung studies) and D7 or D10 (for gut). Longitudinal egg counts in fecal pellets were performed daily from D6 to D10. intestinal worm counts and fecal egg counts were measured on D7 and D10. Matched samples of lung (D4) and gut (D7 and D10) tissue for quantitative PCR were collected and processed as below.

Quantitative PCR. Tissue samples were mechanically homogenized in Trizol using beads and a Precellys24 (Bertin Technologies, Rockville, MD). RNA was extracted with chloroform, isolated with a MagMAX total RNA isolation kit, and reverse transcribed with Superscript II (Life Technologies, Grand Island, NY). Gene expression was measured by quantitative PCR amplification of complementary DNA using SYBR Green on an ABI Prism 7900HT Sequence Detection System (all from Life Technologies) and normalized to RPLP2. Real-time PCR

Table 1 Primer sequences

\begin{tabular}{|c|c|c|c|}
\hline Gene symbol & Common name(s) & Forward primer sequence $\left(5^{\prime}-3^{\prime}\right)$ & Reverse primer sequence $\left(5^{\prime}-3^{\prime}\right)$ \\
\hline $\operatorname{Arg} 2$ & Arginase 2 & TCCTCCACGGGCAAATTCC & GCTGGACCATATTCCACTCCTA \\
\hline CCL2 & CCL2, MCP 1 & AGGTGTCCCAAAGAAGCTGTA & ATGTCTGGACCCATTCCTTCT \\
\hline CCL11 & CCL11, Eotaxin & GAATCACCAACAACAGATGCAC & ATCCTGGACCCACTTCTTCTT \\
\hline Сhiß/3 & Ym1 & CATGAGCAAGACTTGCGTGAC & GGTCCAAACTTCCATCCTCCA \\
\hline Clca3 & Gob5 & CATCGCCATAGACCACGACG & TTCCAGCTCTCGGGAATCAAA \\
\hline Ifng & $\mathrm{IFN}-\gamma$ & AGAGCCAGATTATCTCTITCTACCTCAG & CCTIITCGCCTTGCTGTTG \\
\hline $1 / 5$ & $\| \mathrm{L}-5$ & TGACAAGCAATGAGACGATGAGG & ACCCCCACGGACAGTTTGATTC \\
\hline $1 / 10$ & IL-10 & ATGCTGCCTGCTCTTACTGACTG & CCCAAGTAACCCTTAAAGTCCTGC \\
\hline II13Ra2 & IL-13 receptor $\alpha 2$ & GGAAAGGAGGACAAAGAGGTC & GATTTAGTGTGCTGAAAGCTCTACTC \\
\hline Muc5ac & Muc5AC & CAGGACTCTCTGAAATCGTACCA & AAGGCTCGTACCACAGGGA \\
\hline Nos2 & iNOS & TGCCCCTTCAATGGTTGGTA & ACTGGAGGGACCAGCCAAAT \\
\hline Retnla & RELM $\alpha$, Fizz1 & СССТCCACTGTAACGAAGACTC & CACACCCAGTAGCAGTCATCC \\
\hline Retnlb & RELM $\beta$, Fizz2 & CGTCTCCCTITCCCACTG & CAGGAGATCGTCTTAGGCTCTT \\
\hline Rp/p2 & RPLP2 & TACGTCGCCTCTTACCTGCT & GACCTTGTTGAGCCGATCAT \\
\hline
\end{tabular}


data were analyzed using the comparative Ct method using SAGE software (https://sage.niaid.nih.gov). All experiments used intronspanning primers validated by dissociation curves (see Table 1).

Chemokine ELISA. Snap-frozen lung lobes were homogenized in RIPA lysis buffer (Santa Cruz Biotechnology, Dallas, TX) using Precellys24 (Bertin Technologies). Protein concentration of the clarified lysates were measured by absorbance at $280 \mathrm{~nm}$ using Nanodrop (Waltham, MA). Concentrations of CCL1, CCL2, and CCL22 were measured in the lysate using respective enzyme-linked immunosorbent assay (ELISA) kits (R\&D Systems, Minneapolis, MN) as per the manufacturer's protocol.

Lung leukocyte isolation. Lung leukocytes were isolated by digesting PBS perfused matched lung lobes after mincing in collagenase IV (100 $\mathrm{U} \mathrm{ml}^{-1}$ in RPMI) for $60 \mathrm{~min}$ rocking at $37^{\circ} \mathrm{C}$, crushing through a $100 \mu \mathrm{M}$ cell strainer, underlaying with isotonic 36\% Percoll (GE Healthcare, Piscataway, NJ), and centrifuging for $20 \mathrm{~min}$ at $350 \mathrm{~g}$. Erythrocytes were eliminated using ACK lysis buffer (Quality Biological, Gaithersburg, MD). Lung leukocyte numbers were determined using Trypan blue and a Cellometer Auto T4 (Nexcelom, Lawrence, MA) or hemocytometer.

T-cell restimulation and flow cytometry. To assess intracellular cytokines, leukocytes were stimulated for $4 \mathrm{~h}$ with phorbol 12-myristate 13 -acetate $\left(10 \mathrm{ng} \mathrm{ml}^{-1}\right)$, ionomycin $\left(1 \mu \mathrm{g} \mathrm{ml}^{-1}\right)$, and brefeldin A $\left(10 \mu \mathrm{g} \mathrm{ml}^{-1}\right)$. Cells were washed, fixed in $2 \%$ paraformaldehyde, permeabilized with $0.5 \%$ saponin buffer, and stained with antibodies specific for CD4 (Biolegend, San Diego, CA; RM4-5), IFN- $\gamma$ (eBioscience, San Diego, CA; XMG1.2), IL-4 (eBioscience; 11B11), IL13 (eBioscience; eBio13A), and IL-17A (Biolegend; TC11-18H10.1). To assess cell populations and macrophage depletion, leukocytes isolated from lung tissue or $72 \mathrm{~h}$ thioglycollate elicited peritoneal cells $(3 \% \mathrm{w} / \mathrm{v})$ were labeled with a viability dye (Invitrogen, Grand Island, NY; LIVE/DEAD Blue or Aqua), preincubated in $2 \%$ normal rat serum plus unlabeled anti-CD16/32 antibody (eBioscience; 93), and stained with combinations of antibodies specific for: CD11b (Biolegend; M1/ 70), CD11c (BD; HL3), CD45 (Biolegend; 30-F11), CD64 (Biolegend; X54-5/7.1), F4/80 (Biolegend; BM8), Gr-1 (Biolegend; RB6-8C5), Ly6C (Biolegend; HK1.4), Ly6G (BD; 1A8), MHCII I-Ab (BD; AF6120.1) or I-A/E (Biolegend; M5/114.15.2), Siglec-F (BD; E50-2440), and T-cell receptor- $\beta$ (eBioscience; H57-597). Flow cytometry was performed using a FACSCanto II or LSRII (BD Biosciences) and data were analyzed using FlowJo (Tree Star, Ashland, OR).

T-cell adoptive transfer. Antigen-presenting cells were prepared from naive $\mathrm{C} 57 \mathrm{BL} / 6$ or $\mathrm{CD} 11 \mathrm{~b}-\mathrm{DTR}$ mice. Spleens were digested in collagenase (100 $\mathrm{U} \mathrm{ml}^{-1}$ in RPMI) for 60 min rocking at $37^{\circ} \mathrm{C}$, mechanically dispersed to generate single-cell suspensions, and treated with ACK lysis buffer to eliminate erythrocytes. Spleen plus peripheral and mesenteric lymph nodes of ovalbumin-specific OT-II T-cell receptor transgenic donor mice were processed similarly, labeled with biotinylated anti-CD4 antibody (eBioscience; GK1.5), and then enriched for $\mathrm{CD}^{+}{ }^{+} \mathrm{T}$ cells by positive selection with MACS LS columns and streptavidin or anti-biotin beads (Miltenyi Biotec, San Diego, CA). Antigen-presenting cells were irradiated with 3,300 rads. To activate OT-II cells in vitro, 10 irradiated antigen-presenting cells per $\mathrm{CD}^{+}$cell were cultured at $37^{\circ} \mathrm{C}$ in RPMI plus $1 \mu \mathrm{g} \mathrm{ml}{ }^{-1}$ ovalbumin 323-339 peptide and 1 or $20 \mu \mathrm{g} \mathrm{ml}^{-1}$ soluble egg antigen. Culture media were supplemented with $10 \mathrm{Uml}^{-1}$ recombinant human IL-2 (NCI repository, Frederick, MD). Recipient mice (CD11bDTR) were primed by IP injection of 5,000 S. mansoni eggs in PBS on D-14 and injected IV with 5,000 live eggs in PBS at D0 to induce lung granulomas. Half the mice were treated with DTX $\left(25 \mathrm{ng} \mathrm{g}^{-1}\right)$ at D3, $\mathrm{D} 4$, and D5. On D6, CD4 ${ }^{+}$T cells $\left(4 \times 10^{6}\right)$ labeled with CFSE $(5 \mu \mathrm{M}$ for $10 \mathrm{~min}$ at room temperature) were injected IV in $300 \mu \mathrm{l}$ of PBS to recipient mice. Mice were killed by pentobarbital overdose plus heparin $24 \mathrm{~h}$ later. The number of CFSE-labeled CD $4^{+}$cells in blood, lung, inguinal lymph nodes, and mediastinal lymph nodes was assessed by flow cytometry.

Bone marrow chimeras. Bone marrow was harvested from the femur and tibia of C57Bl/6, CD11b-DTR, CD11c-DTR, and $\mathrm{CD} 11 \mathrm{~b} / \mathrm{c}-\mathrm{DTR}$ mice, washed, filtered, resuspended in PBS, and injected IV $\left(4 \times 10^{6}\right.$ cells) into lethally irradiated (900 rads) C57Bl/6 recipient mice. Mice were provided antibiotic-containing water (Trimethoprim-sulfa) for 4 weeks and used for experiments 7 weeks after reconstitution.

Statistical analyses. All data were analyzed with Prism (GraphPad Software, La Jolla, CA). Data were considered statistically significant for $P$-values $<0.05$, obtained with a two-tailed $t$-test or MannWhitney $U$-test. Differences are noted as ${ }^{\star} P<0.05$, ${ }^{\star *} P<0.01$, ${ }^{* * *} P<0.001$, or NS (not significant).

SUPPLEMENTARY MATERIAL is linked to the online version of the paper at http://www.nature.com/mi

\section{ACKNOWLEDGMENTS}

We express our sincere appreciation and thanks to all of our colleagues, past and present, for their guidance and support. L.A.B. is supported by a Marie Curie international outgoing fellowship from the European Union Framework Programme 7. T.A.W. is supported by the Intramural Research Program of the NIH/NIAID.

\section{DISCLOSURE}

The authors declared no conflict of interest.

c) 2016 Society for Mucosal Immunology

\section{REFERENCES}

1. Holgate, S.T. Innate and adaptive immune responses in asthma. Nat. Med. 18, 673-683 (2012).

2. Bamias, G., Kaltsa, G. \& Ladas, S.D. Cytokines in the pathogenesis of ulcerative colitis. Discov. Med. 11, 459-467 (2011).

3. Anthony, R.M., Rutitzky, L.I., Urban, J.F. Jr, Stadecker, M.J. \& Gause, W.C. Protective immune mechanisms in helminth infection. Nat. Rev. Immunol. 7, 975-987 (2007).

4. Jakubzick, C. et al. Minimal differentiation of classical monocytes as they survey steady-state tissues and transport antigen to lymph nodes. Immunity 39, 599-610 (2013).

5. Hashimoto, D. et al. Tissue-resident macrophages self-maintain locally throughout adult life with minimal contribution from circulating monocytes. Immunity 38, 792-804 (2013).

6. Guilliams, M. et al. Alveolar macrophages develop from fetal monocytes that differentiate into long-lived cells in the first week of life via GM-CSF. J. Exp. Med. 210, 1977-1992 (2013).

7. Geissmann, F. et al. Development of monocytes, macrophages, and dendritic cells. Science 327, 656-661 (2010).

8. Jenkins, S.J. et al. Local macrophage proliferation, rather than recruitment from the blood, is a signature of $\mathrm{TH}_{2}$ inflammation. Science 332 , 1284-1288 (2011).

9. Pittet, M.J., Nahrendorf, M. \& Swirski, F.K. The journey from stem cell to macrophage. Ann. NY Acad. Sci. 1319, 1-18 (2014).

10. Chen, F. et al. An essential role for $\mathrm{TH}$-type responses in limiting acute tissue damage during experimental helminth infection. Nat. Med. 18, 260-266 (2012).

11. Perrigoue, J.G. et al. MHC class II-dependent basophil-CD4 ${ }^{+}$T cell interactions promote $\mathrm{T}(\mathrm{H}) 2$ cytokine-dependent immunity. Nat. Immunol. 10, 697-705 (2009).

12. Zhao, A. et al. Th2 cytokine-induced alterations in intestinal smooth muscle function depend on alternatively activated macrophages. Gastroenterology 135, 217-225 (2008).

13. Duffield, J.S. et al. Selective depletion of macrophages reveals distinct, opposing roles during liver injury and repair. J. Clin. Invest. 115, 56-65 (2005). 
14. Cailhier, J.F. et al. Conditional macrophage ablation demonstrates that resident macrophages initiate acute peritoneal inflammation. J. Immunol. 174, 2336-2342 (2005).

15. Lin, S.L., Castano, A.P., Nowlin, B.T., Lupher, M.L. Jr \& Duffield, J.S. Bone marrow Ly6Chigh monocytes are selectively recruited to injured kidney and differentiate into functionally distinct populations. J. Immunol. 183, 6733-6743 (2009).

16. Medoff, B.D. et al. CD11b + myeloid cells are the key mediators of Th2 cell homing into the airway in allergic inflammation. J. Immunol. 182, 623-635 (2009).

17. Dhaliwal, K. et al. Monocytes control second-phase neutrophil emigration in established lipopolysaccharide-induced murine lung injury. Am. J. Respir. Crit. Care Med. 186, 514-524 (2012).

18. Crapster-Pregont, M., Yeo, J., Sanchez, R.L. \& Kuperman, D.A. Dendritic cells and alveolar macrophages mediate IL-13-induced airway inflammation and chemokine production. J. Allergy Clin. Immunol. 129, 1621-1627 (2012).

19. Lee, S. et al. Distinct macrophage phenotypes contribute to kidney injury and repair. J. Am. Soc. Nephrol. 22, 317-326 (2011).

20. Wynn, T.A., Eltoum, I., Oswald, I.P., Cheever, A.W. \& Sher, A. Endogenous interleukin 12 (IL-12) regulates granuloma formation induced by eggs of Schistosoma mansoni and exogenous IL-12 both inhibits and prophylactically immunizes against egg pathology. J. Exp. Med. 179, 1551-1561 (1994).

21. Lewis, F. Schistosomiasis. Curr. Protoc. Immunol. Chapter 19, Unit 19.11 (2001).

22. Hesse, M. et al. The pathogenesis of schistosomiasis is controlled by cooperating IL-10-producing innate effector and regulatory $\mathrm{T}$ cells. J. Immunol. 172, 3157-3166 (2004).

23. Hesse, M., Cheever, A.W., Jankovic, \& Wynn, T.A. NOS-2 mediates the protective anti-inflammatory and antifibrotic effects of the Th1-inducing adjuvant, IL-12, in a Th2 model of granulomatous disease. Am. J. Pathol. 157, 945-955 (2000).

24. Chiaramonte, M.G., Donaldson, D.D., Cheever, A.W. \& Wynn, T.A. An IL-13 inhibitor blocks the development of hepatic fibrosis during a T-helper type 2-dominated inflammatory response. J. Clin. Invest. 104, 777-785 (1999).

25. Chiaramonte, M.G., Cheever, A.W., Malley, J.D., Donaldson, D.D. \& Wynn, T.A. Studies of murine schistosomiasis reveal interleukin-13 blockade as a treatment for established and progressive liver fibrosis. Hepatology 34 , 273-282 (2001).

26. Fallon, P.G., Richardson, E.J., McKenzie, G.J. \& McKenzie, A.N.J. Schistosome infection of transgenic mice defines distinct and contrasting pathogenic roles for IL-4 and IL-13: IL-13 is a profibrotic agent. J. Immunol. 164, 2585-2591 (2000).

27. Jung, S. et al. In vivo depletion of CD11C + dendritic cells abrogates priming of CD8 + Tcells by exogenous cell-associated antigens. Immunity 17, 211-220 (2002)

28. Padilla, J. et al. IL-13 regulates the immune response to inhaled antigens. J. Immunol. 174, 8097-8105 (2005).

29. Park, M.K. et al. Patterns of chemokine expression in models of Schistosoma mansoni inflammation and infection reveal relationships between type 1 and type 2 responses and chemokines in vivo. Infect. Immun. 69, 6755-6768 (2001).
30. Souza, P.R., Souza, A.L., Negrao-Correa, D., Teixeira, A.L. \& Teixeira, M.M. The role of chemokines in controlling granulomatous inflammation in Schistosoma mansoni infection. Acta Trop. 108, 135-138 (2008).

31. Mantovani, A. etal. The chemokine system in diverse forms of macrophage activation and polarization. Trends Immunol. 25, 677-686 (2004).

32. Jakubzick, C. et al. Role of CCR4 ligands, CCL17 and CCL22, during Schistosoma mansoni egg-induced pulmonary granuloma formation in mice. Am. J. Pathol. 165, 1211-1221 (2004).

33. Plantinga, M. et al. Conventional and monocyte-derived CD11b(+) dendritic cells initiate and maintain $T$ helper 2 cell-mediated immunity to house dust mite allergen. Immunity 38, 322-335 (2013).

34. Camberis, M., Le Gros, G. \& Urban, J. Jr. Animal model of Nippostrongylus brasiliensis and Heligmosomoides polygyrus. Curr. Protoc. Immunol. Chapter 19, Unit 19.12 (2003).

35. Allen, J.E. \& Wynn, T.A. Evolution of Th2 immunity: a rapid repair response to tissue destructive pathogens. PLoS Pathog. 7, e1002003 (2011).

36. Wynn, T.A., Chawla, A. \& Pollard, J.W. Macrophage biology in development, homeostasis and disease. Nature 496, 445-455 (2013).

37. Barron, L. \& Wynn, T.A. Fibrosis is regulated by Th2 and Th17 responses and by dynamic interactions between fibroblasts and macrophages. Am. J. Physiol. Gastrointest. Liver Physiol. 300, G723-G728 (2011).

38. Duffield, J.S., Lupher, M., Thannickal, V.J. \& Wynn, T.A. Host responses in tissue repair and fibrosis. Annu. Rev. Pathol. 8, 241-276 (2013).

39. Murray, P.J. \& Wynn, T.A. Protective and pathogenic functions of macrophage subsets. Nat. Rev. Immunol. 11, 723-737 (2011).

40. Wynn, T.A. \& Barron, L. Macrophages: master regulators of inflammation and fibrosis. Semin. Liver Dis. 30, 245-257 (2010).

41. van Rijt, L.S. et al. In vivo depletion of lung CD11C + dendritic cells during allergen challenge abrogates the characteristic features of asthma. J. Exp. Med. 201, 981-991 (2005).

42. Hammad, H. et al. Inflammatory dendritic cells-not basophils-are necessary and sufficient for induction of Th2 immunity to inhaled house dust mite allergen. J. Exp. Med. 207, 2097-2111 (2010).

43. Girgis, N.M. et al. Ly6Chigh monocytes become alternatively activated macrophages in Schistosome granulomas with help from $\mathrm{CD} 4{ }^{+}$cells. PLoS Pathog. 10, e1004080 (2014).

44. Jakubzick, C. et al. Blood monocyte subsets differentially give rise to CD103 + and CD103- pulmonary dendritic cell populations. J. Immunol. 180, 3019-3027 (2008).

45. Barron, L. \& Wynn, T.A. Macrophage activation governs schistosomiasisinduced inflammation and fibrosis. Eur. J. Immunol. 41, 2509-2514 (2011).

46. Ramalingam, T.R. et al. Unique functions of the type Il interleukin 4 receptor identified in mice lacking the interleukin 13 receptor alpha1 chain. Nat. Immunol. 9, 25-33 (2008).

47. Wilson, M.S. et al. Bleomycin and IL-1beta-mediated pulmonary fibrosis is IL-17A dependent. J. Exp. Med. 207, 535-552 (2010).

48. Bieren, J.E.V. et al. Antibodies trap tissue migrating helminth larvae and prevent tissue damage by driving $\mathrm{IL}-4 \mathrm{R}$ alpha-independent alternative differentiation of macrophages. PLoS Pathog. 9, e1003771 (2013).

49. Wynn, T.A. et al. Quantitative assessment of macrophage functions in repair and fibrosis. Curr. Protoc. Immunol. Chapter 14, Unit14.22 (2011). 